\title{
Functionalization of
} [2.2]Paracyclophanes via a Reductive Sulfanylation Reaction

Damien Deschamps, ${ }^{a}$ Jean-François Lohier, ${ }^{a}$ Christopher J. Richards, ${ }^{\text {b }}$ Annie-Claude Gaumont ${ }^{\text {a }}$ and Stéphane Perrio*,a

${ }^{a}$ Normandie Univ, ENSICAEN, UNICAEN, CNRS, LCMT, 14000 Caen,

France

${ }^{b}$ School of Chemistry, University of East Anglia, Norwich Research Park, Norwich, NR4 7TJ, U.K

e-mail: stephane.perrio@ensicaen.fr

$$
\text { Supporting Information }
$$

Synthesis of Sulfides 4: Optimization of the Reaction Conditions

Synthesis of Sulfides 4: Stereochemical Assignment and Transition State

Model

Cross-experiments and Chemoselectivity

X-Ray Crystal Structures

Copies of HPLC Chromatographs 
I - Synthesis of Sulfides 4: Optimization of the Reaction Conditions

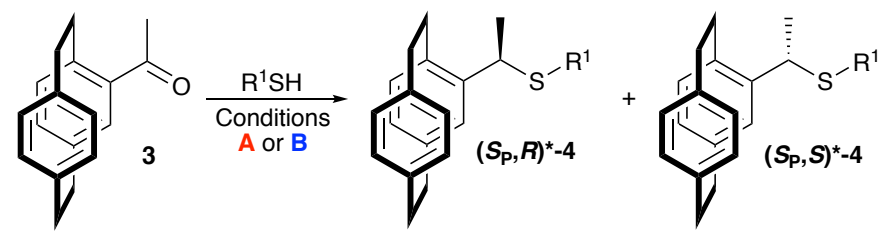

A: $\mathrm{BF}_{3} \cdot \mathrm{OEt}_{2}$ (1.1 equiv), $\mathrm{Et}_{3} \mathrm{SiH}$ (1.5 equiv), $\mathrm{CH}_{2} \mathrm{Cl}_{2}(0,25 \mathrm{M})$

B: $\mathrm{F}_{3} \mathrm{CCO}_{2} \mathrm{H}$ (x equiv), $\mathrm{BH}_{3} \cdot \mathrm{THF}$ (1.1 equiv), $\mathrm{CH}_{2} \mathrm{Cl}_{2}(0,25 \mathrm{M})$

\begin{tabular}{ccccccc}
\hline Entry & $\mathrm{R}^{1}$ & Product & Conditions & $\begin{array}{c}\mathrm{F}_{3} \mathrm{CCO}_{2} \mathrm{H} \\
(\text { equiv) }\end{array}$ & Yield $(\%)$ & $(S \mathrm{P}, R)^{*} /(S \mathrm{P}, S)^{* a}$ \\
\hline 1 & $\mathrm{Ph}$ & $\mathbf{4 a}$ & $\mathbf{A}$ & - & $46^{b}$ & $82: 18$ \\
2 & $\mathrm{Ph}$ & $\mathbf{4 a}$ & $\mathbf{B}^{c}$ & 15 & $59^{b}$ & $83: 17$ \\
3 & $\mathrm{Ph}$ & $\mathbf{4 a}$ & $\mathbf{B}$ & 5 & $88^{d}$ & $93: 7$ \\
4 & $\mathrm{Ph}$ & $\mathbf{4 a}$ & $\mathbf{B}$ & $5^{e}$ & $80^{d}$ & $91: 9$ \\
5 & $t-\mathrm{Bu}$ & $\mathbf{4 b}$ & $\mathbf{B}$ & 5 & $79^{d}$ & $85: 15$ \\
6 & $\mathrm{i}-\mathrm{Pr}$ & $\mathbf{4 c}$ & $\mathbf{B}$ & 5 & $85^{d}$ & $86: 14$ \\
\hline
\end{tabular}

${ }^{a}$ Determined by analysis of the ${ }^{1} \mathrm{H}$ NMR spectrum. ${ }^{b}$ Crude yield. ${ }^{c}$ Formation of 4-ethyl[2.2]PCP as sideproduct. ${ }^{1}{ }^{d}$ Yield after column chromatography on $\mathrm{SiO}_{2} \cdot{ }^{e}$ Concentration of $1 \mathrm{M}$.

Table S1. Optimization of the sulfanylation reaction with ketone 3

Ibrom, K.; Kohn (né Wentzel),G.; Boeckmann, K.-U.; Kraft, R.; Holba-Schulz, P.; Ernst, L. Org. Lett. 2000, 2, 4111-4113. 


\section{II - Synthesis of Sulfides 4: Stereochemical Assignment and Transition State}

Model

According to a single crystals X-ray analysis (see page 6), an $\left(S_{\mathrm{P}}, R\right)^{*}$ configuration was assigned to the major diastereoisomer of $\mathbf{4 a}$. We noticed that the diastereoisomer $\left(S_{\mathbf{P}}, \boldsymbol{R}\right) * \mathbf{- 4 a}$ was more polar than the other diastereoisomer $\left(S_{\mathbf{P}}, S\right) *-4 a\left(R_{f}\right.$ value of 0.08 versus 0.16 , entries 1 and 2 in Table 2). Furthermore, the doublet of the methyl group attached to the stereogenic carbon centre in the ${ }^{1} \mathrm{H}$ NMR spectrum is significantly more deshielded for the major stereoisomer $\left(S_{\mathbf{P}}, \boldsymbol{R}\right) *-4 a(1.67 \mathrm{ppm}$ versus $1.32 \mathrm{ppm}$, entries 1 and 2$)$. As similar trends were observed for compounds $\mathbf{4 b}$ (entries 3 and 4) and $\mathbf{4 c}$ (entries 5 and 6), a similar $\left(S_{\mathrm{P}}, R\right)^{*}$ configuration was assigned to the major diastereoisomers of $\mathbf{4 b}$ and $\mathbf{4 c}$.
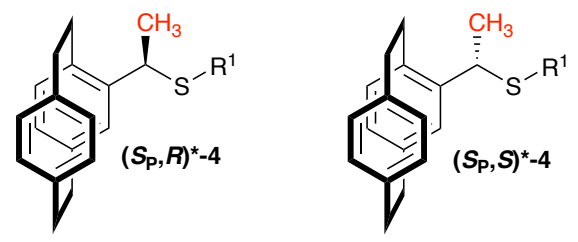

\begin{tabular}{|c|c|c|c|c|}
\hline Entry & Sulfide & $\mathrm{R}^{1}$ & $R_{f}^{a}$ & $\begin{array}{c}{ }^{1} \mathrm{H} \text { NMR: } \\
\delta\left(\mathrm{CH}_{3}\right)(\mathrm{ppm})^{b}\end{array}$ \\
\hline 1 & $\left(S_{\mathrm{p}}, R\right)^{*}-4 \mathrm{a}$ & $\mathrm{Ph}$ & $0.08(90: 10)$ & 1.67 \\
\hline 2 & $\left(S_{\mathrm{p}}, S\right)^{*-4 \mathrm{a}}$ & $\mathrm{Ph}$ & $0.16(90: 10)$ & 1.32 \\
\hline 3 & $\left(S_{\mathrm{p}}, R\right)^{*}-4 \mathrm{~b}$ & $t-\mathrm{Bu}$ & $0.64(80: 20)$ & 1.84 \\
\hline 4 & $\left(S_{\mathrm{p}}, S\right)^{*-4 b}$ & $t-\mathrm{Bu}$ & $0.84(80: 20)$ & 1.45 \\
\hline 5 & $\left(S_{\mathrm{p}}, R\right)^{*}-4 \mathrm{c}$ & $\mathrm{i}-\mathrm{Pr}$ & $0.62(80: 20)$ & 1.75 \\
\hline 6 & $\left(S_{\mathrm{p}}, S\right) *-4 \mathrm{c}$ & i-Pr & $0.82(80: 20)$ & 1.51 \\
\hline
\end{tabular}

${ }^{a} \mathrm{TLC}$ run with pentane/ $\mathrm{CH}_{2} \mathrm{Cl}_{2}$ mixture (ratio under brackets). ${ }^{b}$ Recorded in $\mathrm{CDCl}_{3}$.

Table S2. Analytical data to assign the stereochemistry of diastereoisomers 4

On the basis of the observed diastereoselectivity in favor of the $\left(S_{\mathrm{P}}, R\right)^{*}$ configured product 5, a possible asymmetric induction model is depicted below. The intermediate thionium probably adopts preferentially the conformation $\mathbf{I}$, in which the sulfur moiety is coplanar with the adjacent [2.2]PCP deck and lying away from the $\mathrm{CH}_{2} \mathrm{CH}_{2}$ bridge. The alternative conformation II, in which the thionium is coplanar with benzene ring of the [2.2]PCP but orientated towards the $\mathrm{CH}_{2} \mathrm{CH}_{2}$ group, is disfavored for steric reasons. The 
hydride probably attacks the less hindered $S i$ face of the thionium in I, opposite to the $\mathrm{CH}_{2} \mathrm{CH}_{2}$ group.

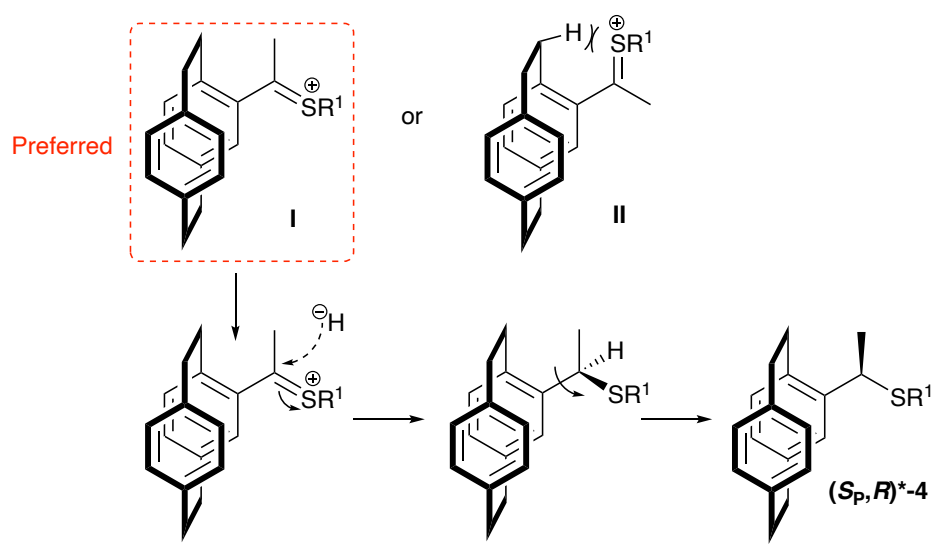

Scheme S1. Induction model for the formation of the major diastereisomer $\left(S_{\mathrm{P}}, R\right) *-4$ 


\section{III - Cross-experiments and Chemoselectivity}

With $\mathrm{BF}_{3} \cdot \mathrm{OEt}_{2} / \mathrm{Et}_{3} \mathrm{SiH}$

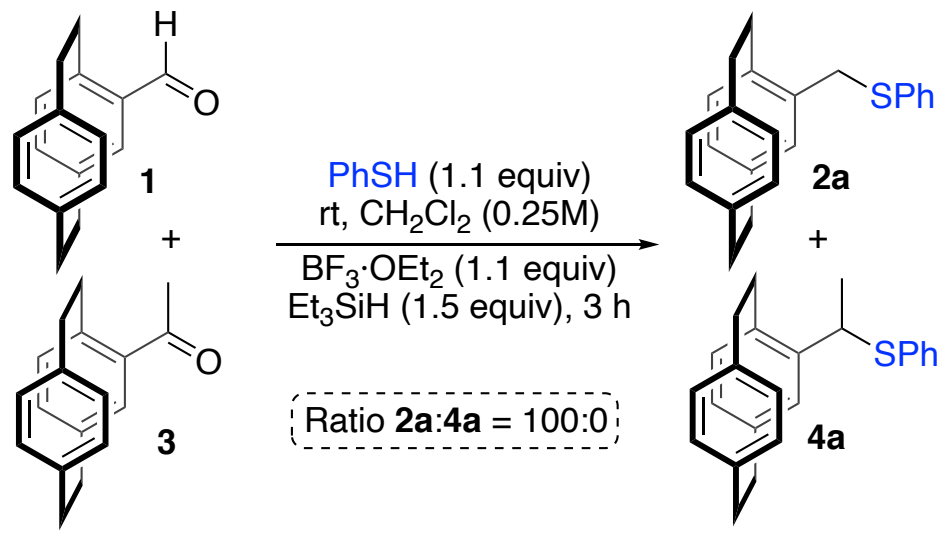

Scheme S2. Cross-experiment with 1 and 3 with the $\mathrm{BF}_{3} \cdot \mathrm{OEt}_{2} / \mathbf{E t}_{3} \mathrm{SiH}$ conditions

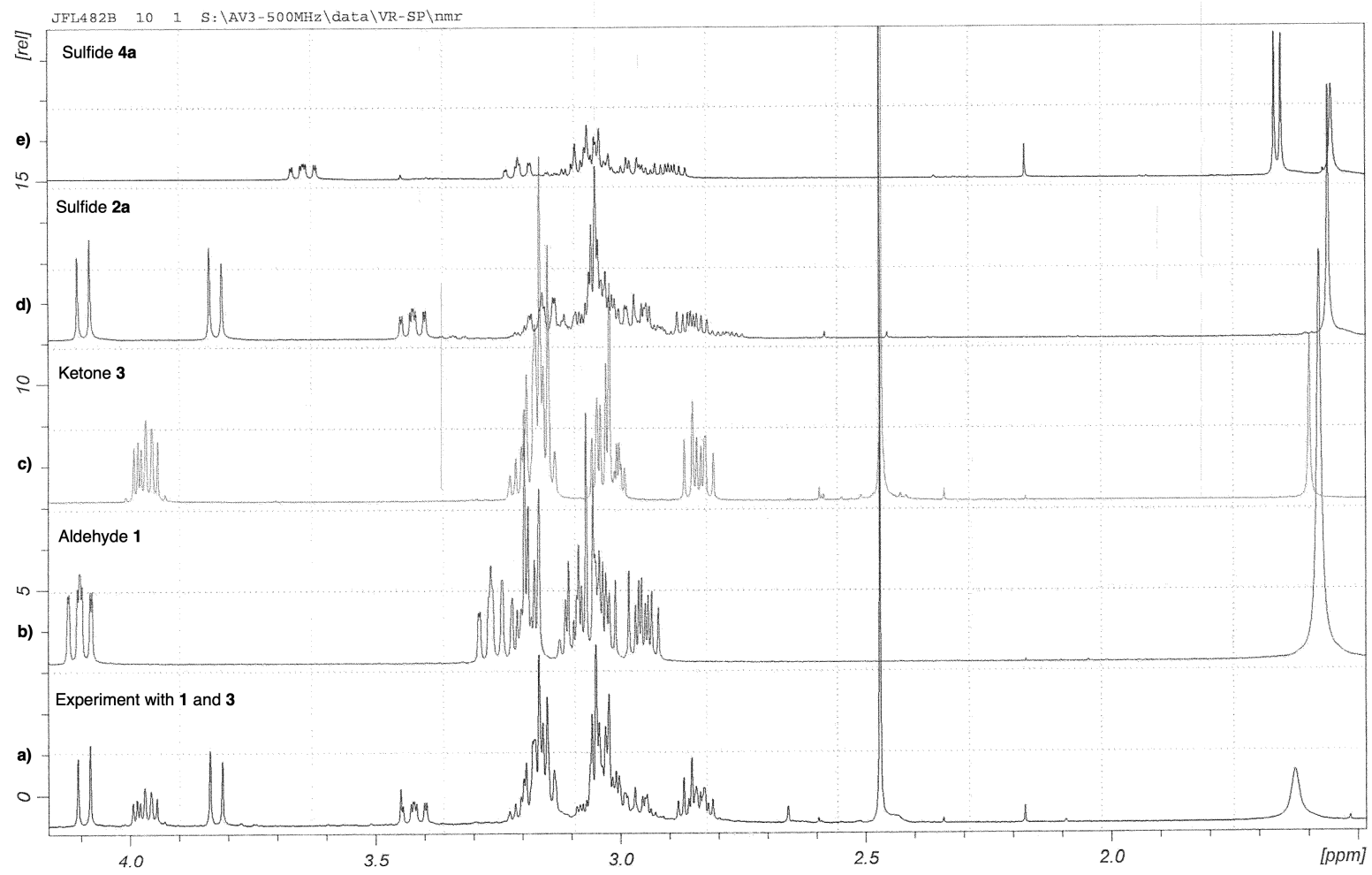

Figure S1. Portions of ${ }^{1} \mathbf{H}$ NMR spectra. a) Experiment with aldehyde $\mathbf{1}$ and ketone $\mathbf{3}$ in the presence of $\mathrm{BF}_{3} \cdot \mathrm{OEt}_{2} / \mathrm{Et}_{3} \mathrm{SiH}$. b) Reactant: Aldehyde 1. c) Reactant: Ketone 3. d) Product: Sulfide 2a. e) Product: Sulfide 4a. 


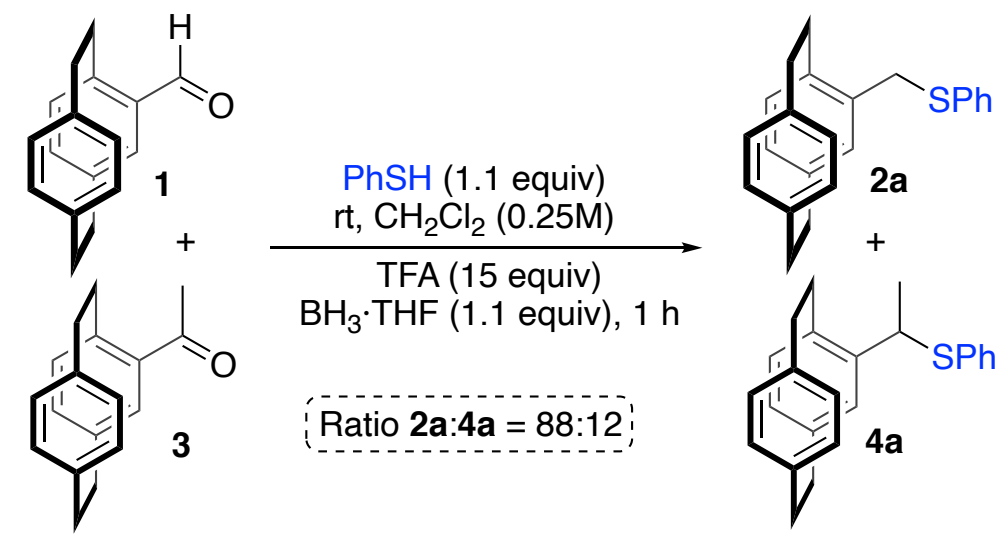

Scheme S3. Cross-experiment with 1 and 3 with the TFA/BH $\cdot$ THF conditions

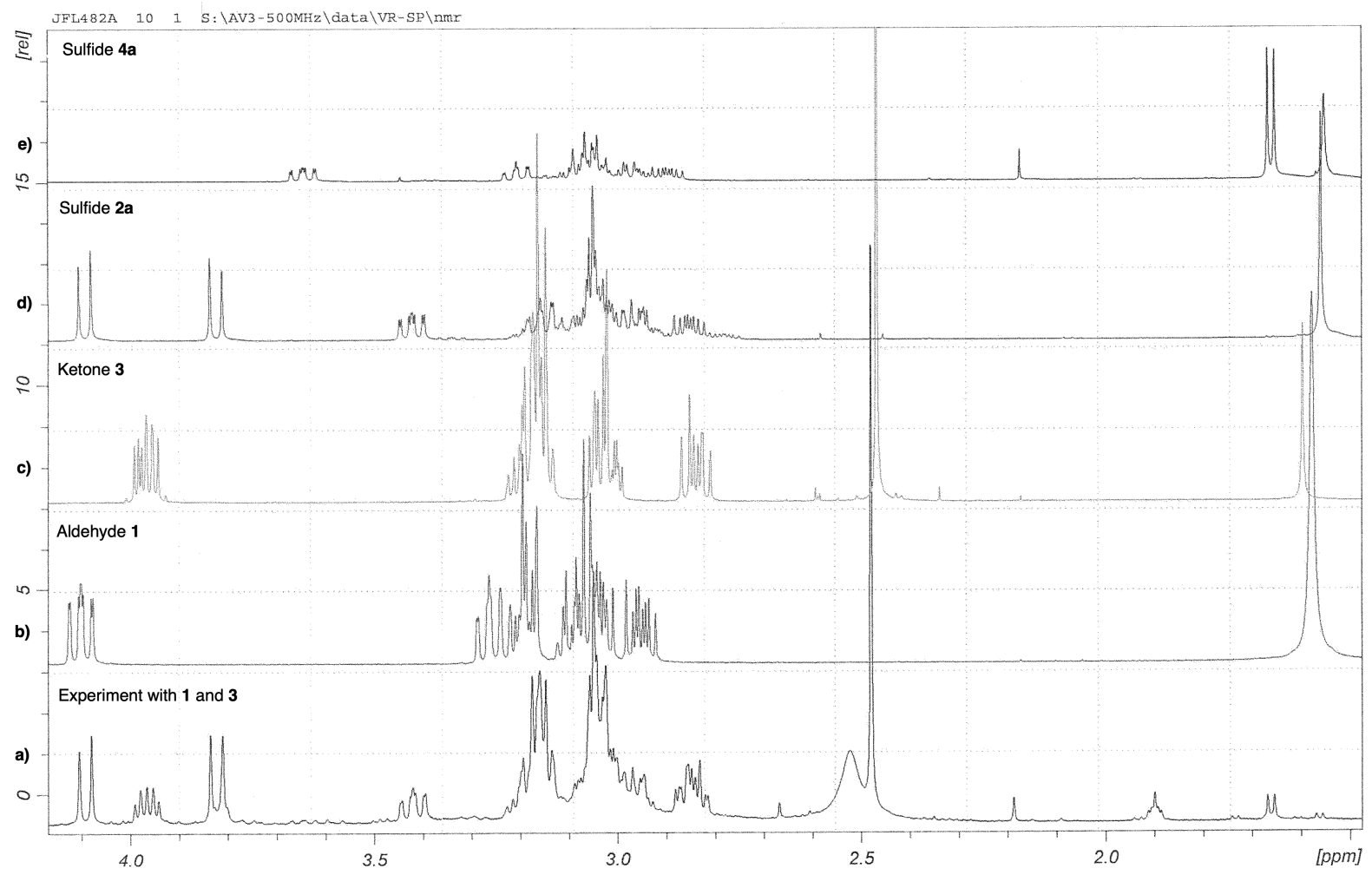

Figure S2. Portions of ${ }^{1} \mathbf{H}$ NMR spectra. a) Experiment with aldehyde $\mathbf{1}$ and ketone $\mathbf{3}$ in the presence of $\mathrm{TFA} / \mathrm{BH}_{3} \cdot \mathrm{THF}$. b) Reactant: Aldehyde 1. c) Reactant: Ketone 3. d) Product: Sulfide 2a. e) Product: Sulfide 4a. 


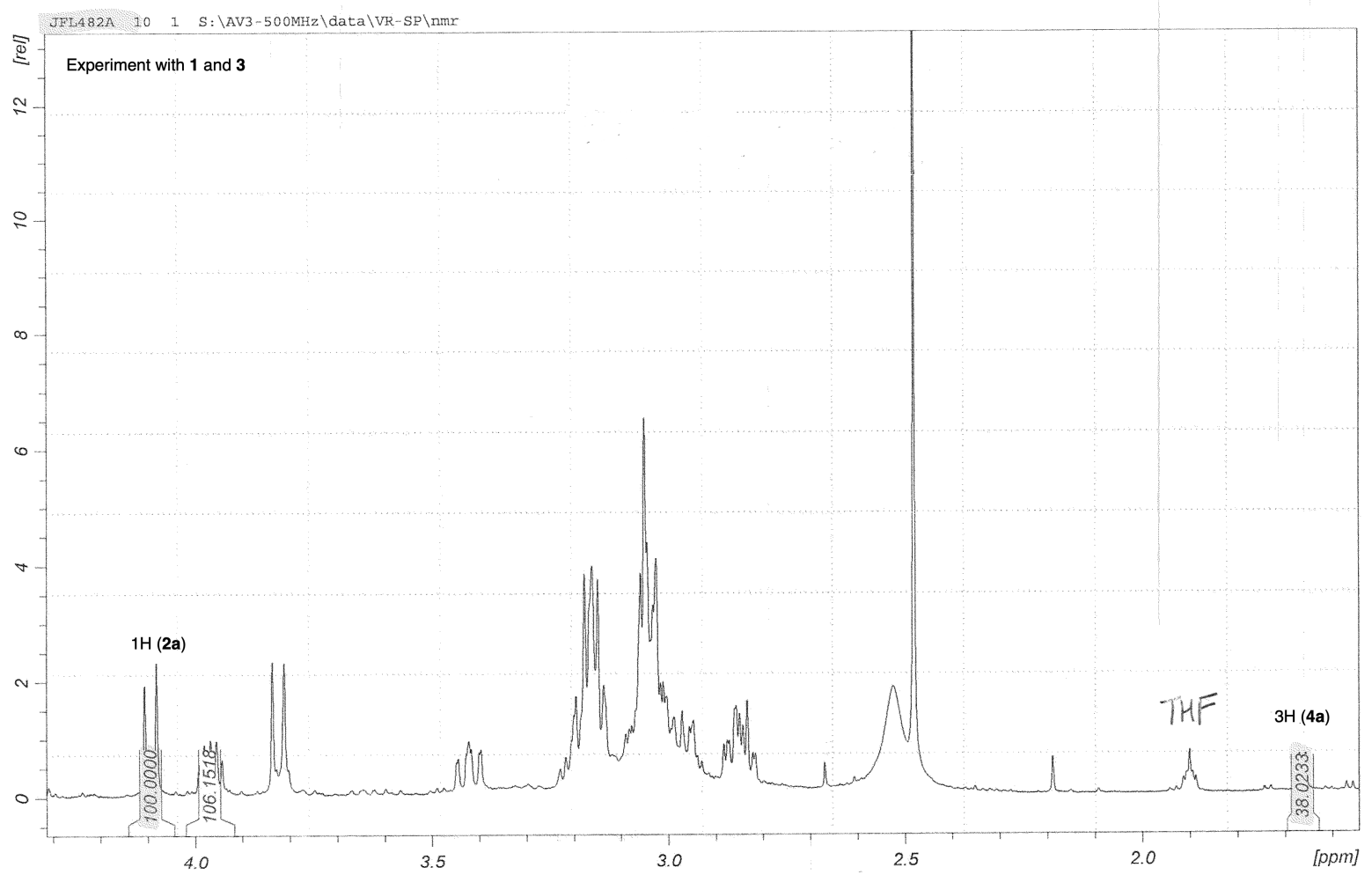

Figure S3. Portions of the ${ }^{1} \mathrm{H}$ NMR spectrum for the determination of the ratio of 2 a and $4 \mathrm{a}$ in the reaction in the presence of $\mathrm{TFA} / \mathrm{BH}_{3} \cdot \mathrm{THF}$. 


\section{4-(Phenylsulfanyl)methyl[2.2]paracyclophane (2a)}

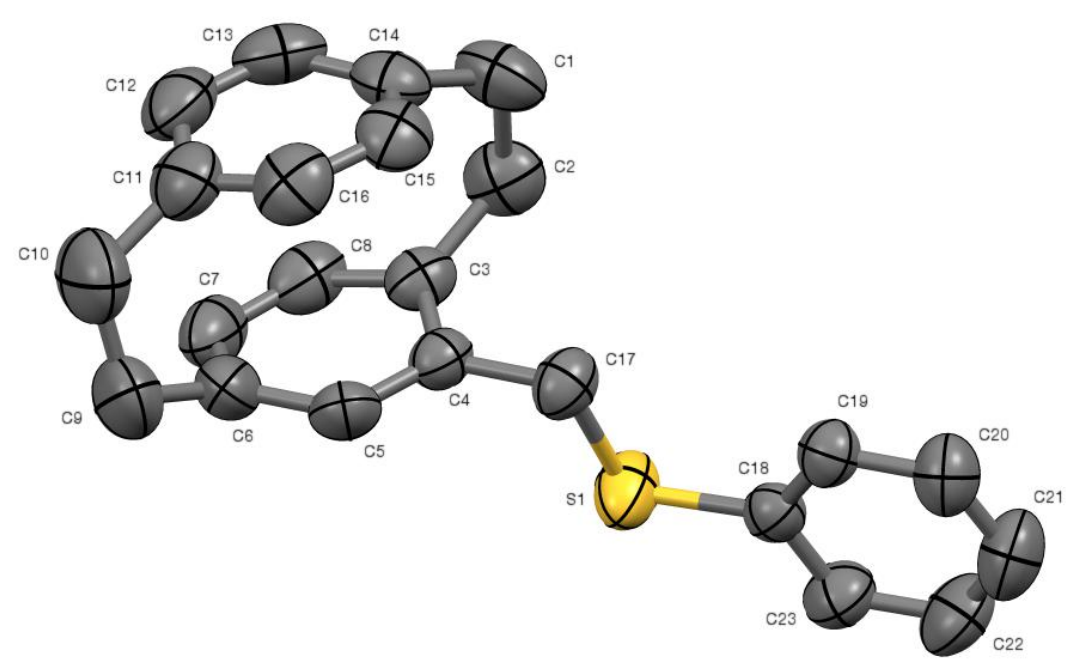

Figure S4. Molecular structure of 2a. Hydrogen atoms are omitted for clarity. Displacement ellipsoids are drawn at the $50 \%$ probability level.

Single crystals of thioether 2a suitable for X-ray crystallographic analysis were obtained by slow diffusion of pentane in $\mathrm{CH}_{2} \mathrm{Cl}_{2}$ solution. X-ray diffraction experiments were performed at $291 \mathrm{~K}$ with graphite-monochromatized Mo $\mathrm{K}_{\alpha}$ radiation $(\lambda=0.71073 \AA)$ on a Bruker-Nonius Kappa CCD area detector diffractometer. Formula $\mathrm{C}_{23} \mathrm{H}_{22} \mathrm{~S}$, formula weight 330.47, crystal system orthorhombic, space group Pbca, $a=8.6484(2) \AA, b=10.3872(3) \AA, c$ $=40.4361(11) \AA, \alpha=\beta=\gamma=90^{\circ}, \mathrm{V}=3632.48(17) \AA^{3}, \mathrm{Z}=8$, calculated density $=1.209$ $\mathrm{g} / \mathrm{cm}^{3}, \mu=0.178 \mathrm{~mm}^{-1}, \mathrm{R}_{\mathrm{int}}=0.0415, \mathrm{R}\left[F^{2}>2 \sigma\left(F^{2}\right)\right]=0.0544, \mathrm{wR}\left(F^{2}\right)=0.1308, \mathrm{GOF}=1.104$, 217 parameters, final difference map within 0.291 and $-0.321 \mathrm{e}^{-3}$. The structure was solved using direct methods and refined by full-matrix least-squares analysis on $F^{2}$. Selected bond lengths $(\AA)$, angles (deg) and dihedral angles (deg): S1-C18 1.758(2), S1-C17 1.807(2), C17-S1-C18 105.61(11), C4-C17-S1 105.76(15). Program(s) used to solve structure: SHELXS-2014/5. Program(s) used to refine structure: SHELXL-2017/1. Software used to prepare material for publication: SHELXTL-97. CCDC 1909742 contains the supplementary crystallographic data for this paper. These data can be obtained free of charge from The Cambridge Crystallographic Data Center via www.ccdc.cam.ac.uk/data_request/cif. 


\section{$\left(S_{\mathrm{P}}, \boldsymbol{R}\right)^{*}-4-\left[1\right.$ '-(Phenylsulfanyl)ethyl]-[2.2]paracyclophane $\left(\left(S_{\mathrm{P}}, \boldsymbol{R}\right)^{*}-4 \mathrm{a}\right)$}

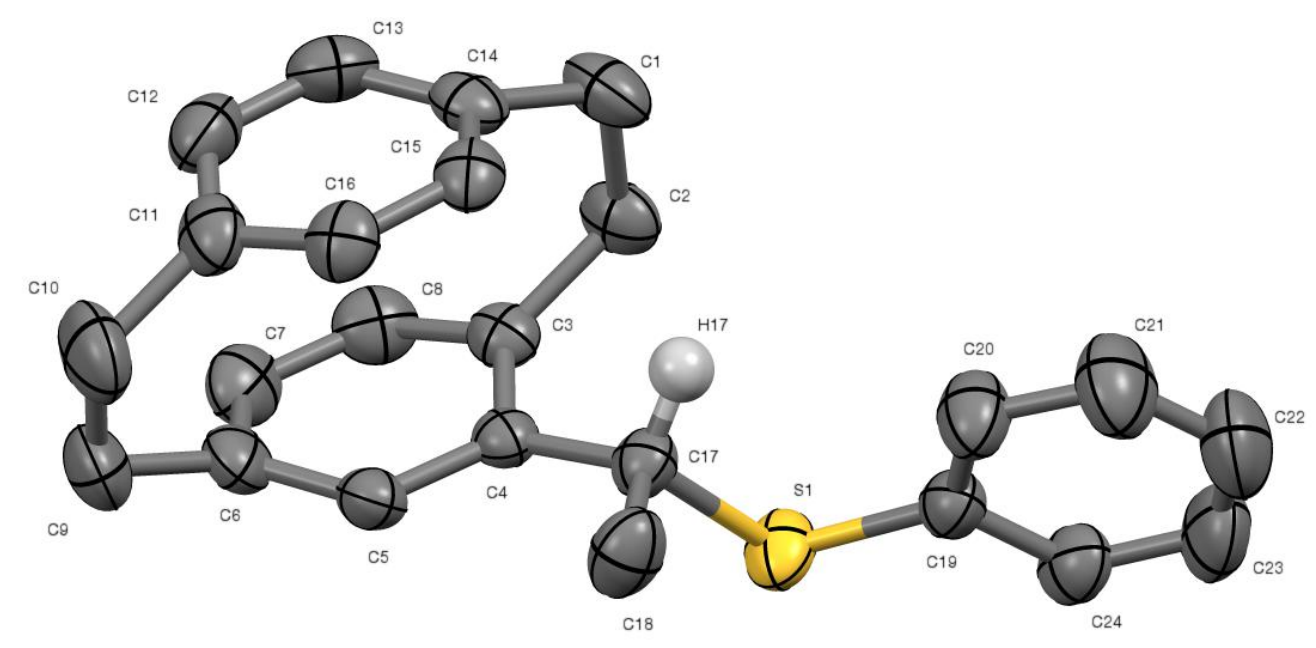

Figure S5. Molecular structure of $\left(S_{\mathbf{P}}, \boldsymbol{R}\right) *-4 a$. Hydrogen atoms, except the stereochemically relevant $\mathrm{H} 17$ hydrogen atom, are omitted for clarity. Displacement ellipsoids are drawn at the $50 \%$ probability level.

Single crystals of $\left(S_{\mathrm{P}}, R\right)^{*}$ configured sulfide $\left(\boldsymbol{S}_{\mathbf{P}}, \boldsymbol{R}\right) *-4 a$ suitable for X-ray crystallographic analysis were obtained by slow diffusion of pentane in $\mathrm{CH}_{2} \mathrm{Cl}_{2}$ solution. Xray diffraction experiments were performed at $291 \mathrm{~K}$ with graphite-monochromatized Mo $\mathrm{K}_{\alpha}$ radiation $(\lambda=0.71073 \AA)$ on a Bruker-Nonius Kappa CCD area detector diffractometer. Formula $\mathrm{C}_{24} \mathrm{H}_{24} \mathrm{~S}$, formula weight 344.49 , crystal system monoclinic, space group $P 21 / \mathrm{c}, a=$ 9.3450(3) $\AA, b=17.2754(5) \AA, c=11.6767(4) \AA, \alpha=90^{\circ} \beta=99.0810(10)^{\circ} \gamma=90^{\circ}, \mathrm{V}=$ 1861.44(10) $\AA^{3}, Z=4$, calculated density $=1.229 \mathrm{~g} / \mathrm{cm}^{3}, \mu=0.177 \mathrm{~mm}^{-1}, R_{\text {int }}=0.0267$, $\mathrm{R}\left[F^{2}>2 \sigma\left(F^{2}\right)\right]=0.0419, \mathrm{wR}\left(F^{2}\right)=0.1130, \mathrm{GOF}=1.053,322$ parameters, final difference map within 0.204 and $-0.228 \mathrm{e}^{-3}$. The structure was solved using direct methods and refined by full-matrix least-squares analysis on $F^{2}$. Selected bond lengths $(\AA)$, angles (deg) and dihedral angles (deg): S1-C19 1.767(1), S1-C17 1.832(1), C4-C17-S1-C19 155.83(8), C5-C4-C17C18 -2.1(2). Program(s) used to solve structure: SHELXS-97. Program(s) used to refine structure: SHELXL-97. Software used to prepare material for publication: SHELXTL-97. CCDC 1909741 contains the supplementary crystallographic data for this paper. These data can be obtained free of charge from The Cambridge Crystallographic Data Center via www.ccdc.cam.ac.uk/data_request/cif. 
$\left(R_{P}\right)-[2.2]$ Paracyclophan-4-ylmethanethiol (( $\left.\left.R_{P}\right)-5\right)$

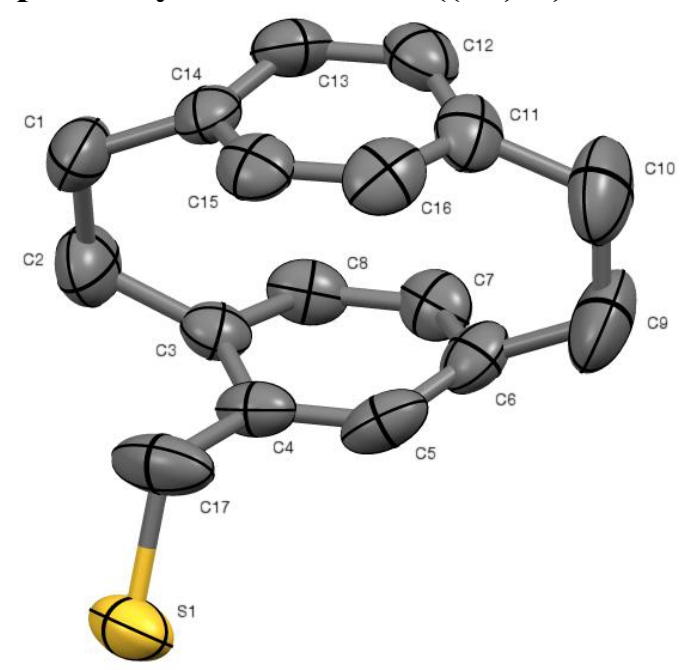

Figure S6. Molecular structure of $\left(\boldsymbol{R}_{\mathbf{P}}\right)$-5. Hydrogen atoms are omitted for clarity. Displacement ellipsoids are drawn at the $50 \%$ probability level.

Single crystals of $\left(R_{\mathrm{P}}\right)-[2.2]$ paracyclophan-4-ylmethanethiol $\left(\boldsymbol{R}_{\mathbf{P}}\right)-\mathbf{5}$ suitable for X-ray crystallographic analysis were obtained by slow diffusion of pentane in $\mathrm{CH}_{2} \mathrm{Cl}_{2}$ solution. Xray diffraction experiments were performed at $291 \mathrm{~K}$ with graphite-monochromatized Mo $\mathrm{K}_{\alpha}$ radiation $(\lambda=0.71073 \AA)$ on a Bruker-Nonius Kappa CCD area detector diffractometer. Formula $\mathrm{C}_{17} \mathrm{H}_{19} \mathrm{~S}$, formula weight 255.38 , crystal system orthorhombic, space group $P 2{ }_{1}{ }_{1} 2_{1}$, $a=7.5508(2) \AA, b=12.5915(3) \AA, c=14.3682(3) \AA, \alpha=\beta=\gamma=90^{\circ}, \mathrm{V}=1366.07(6) \AA^{3}, \mathrm{Z}$ $=4$, calculated density $=1.242 \mathrm{~g} / \mathrm{cm}^{3}, \mu=0.216 \mathrm{~mm}^{-1}, \mathrm{R}_{\text {int }}=0.0169, \mathrm{R}\left[F^{2}>2 \sigma\left(F^{2}\right)\right]=0.0543$, $\mathrm{w} \mathrm{R}\left(F^{2}\right)=0.1617, \mathrm{GOF}=1.033,164$ parameters, final difference map within 0.379 and -0.491 $\mathrm{e} \AA^{-3}$. The structure was solved using direct methods and refined by full-matrix least-squares analysis on $F^{2}$. Selected bond lengths $(\AA)$, angles (deg) and dihedral angles (deg): S1-C17 1.796(3), C4-C17 1.515(3), C4-C17-S1 110.4(2). Program(s) used to solve structure: SHELXS-97. Program(s) used to refine structure: SHELXL-97. Software used to prepare material for publication: SHELXTL-97. CCDC 1909740 contains the supplementary crystallographic data for this paper. These data can be obtained free of charge from The Cambridge Crystallographic Data Center via www.ccdc.cam.ac.uk/data request/cif. 


\section{V - Copies of HPLC Chromatographs}

a) HPLC trace for ( \pm )-2e on a Diacel Chiralpak IA column (i.d., $4.6 \mathrm{~mm}$; eluent, 60:40 $\mathrm{n}$ heptane/EtOH; flow rate, $1.0 \mathrm{~mL} / \mathrm{min}$ ).

b) HPLC trace for the combined fractions of $\left(\boldsymbol{R}_{\mathbf{P}}\right)-\mathbf{2 e}$ (i.d., $4.6 \mathrm{~mm}$; eluent, 60:40 $\mathrm{n}$ heptane/EtOH; flow rate, $1.0 \mathrm{~mL} / \mathrm{min}$ ).

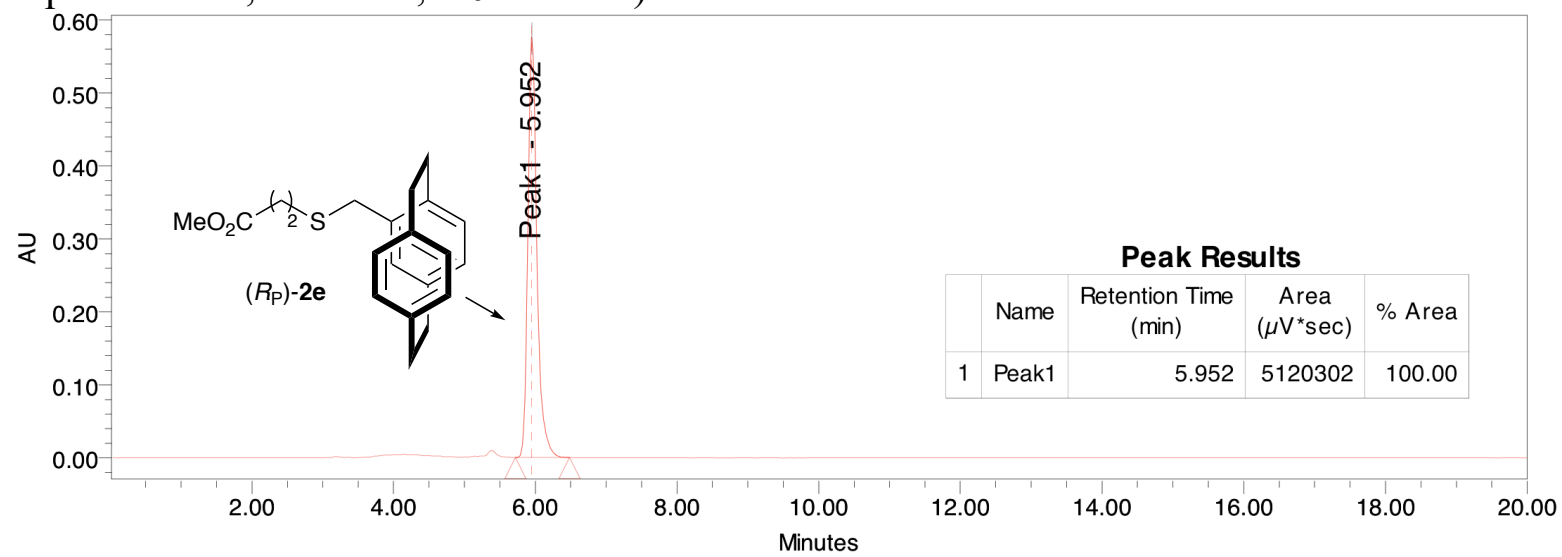

c) HPLC trace for the combined fractions of $\left(S_{\mathbf{P}}\right)-2 e$ (i.d., $4.6 \mathrm{~mm}$; eluent, 60:40 $\mathrm{n}$ heptane $/ \mathrm{EtOH}$; flow rate, $1.0 \mathrm{~mL} / \mathrm{min}$ ).

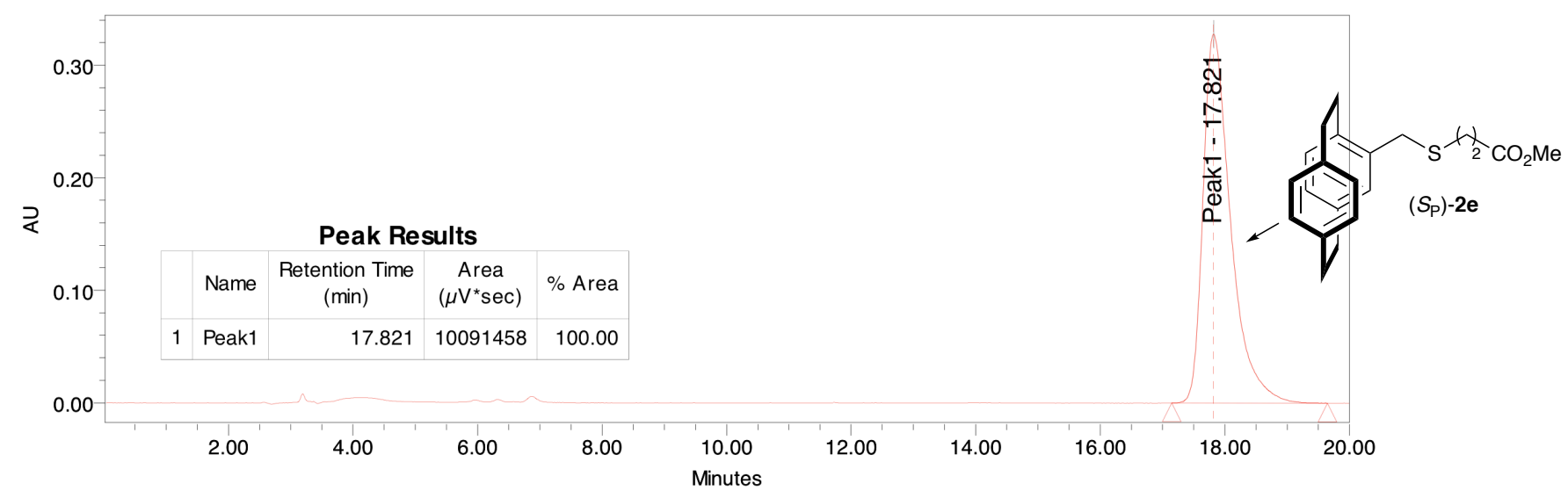




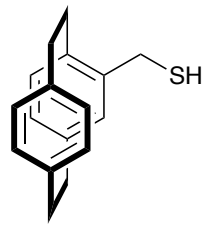

$( \pm)-5$

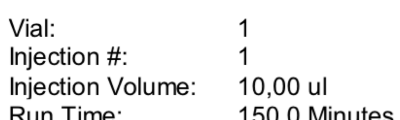

Run Time: 150,0 Minutes

\section{NFORMATION}

$\begin{array}{ll}\text { Acquired By: } & \text { System } \\ \text { Sample Set Name: } & \text { m } \\ \text { Acq. Method Set: } & \text { PM IA 1ml } 8020 \text { hept EtOH 20dc } \\ \text { Processing Method: } & \text { proc } \\ \text { Channel Name: } & 210,0 \mathrm{~nm} \\ \text { Proc. Chnl. Descr.: } & \text { PDA 210,0 nm }\end{array}$

Spectrum Index Plot

Peak1 - 4,801
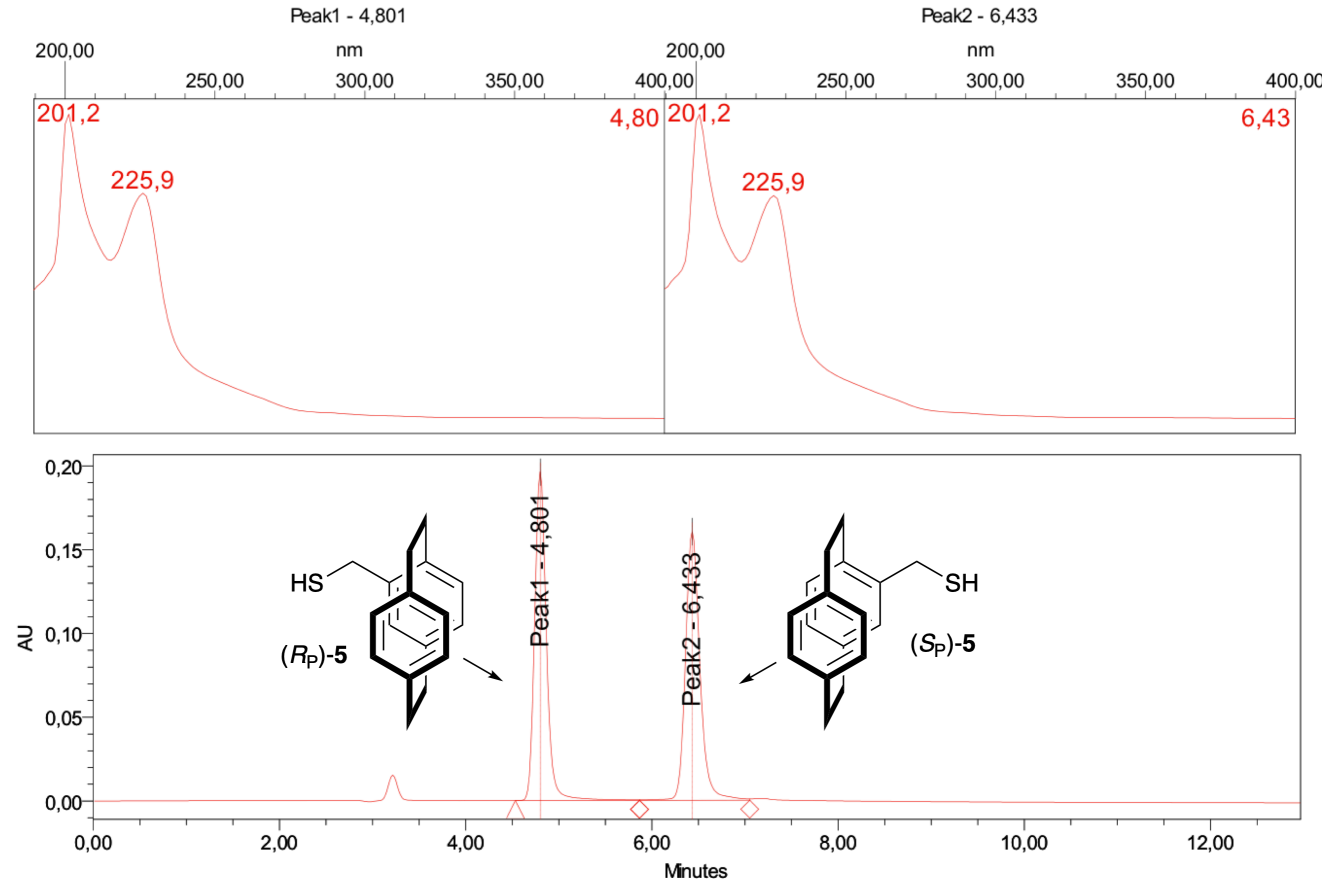

Peak Results

Name RT Area \% Area

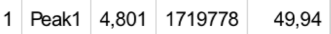

\begin{tabular}{l|l|l|l|l|}
2 & Peak2 & 6,433 & 1723742 & 50,06 \\
\hline
\end{tabular} 


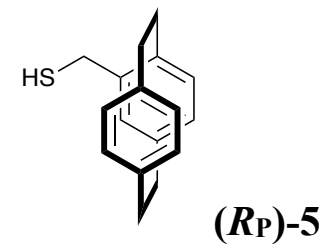

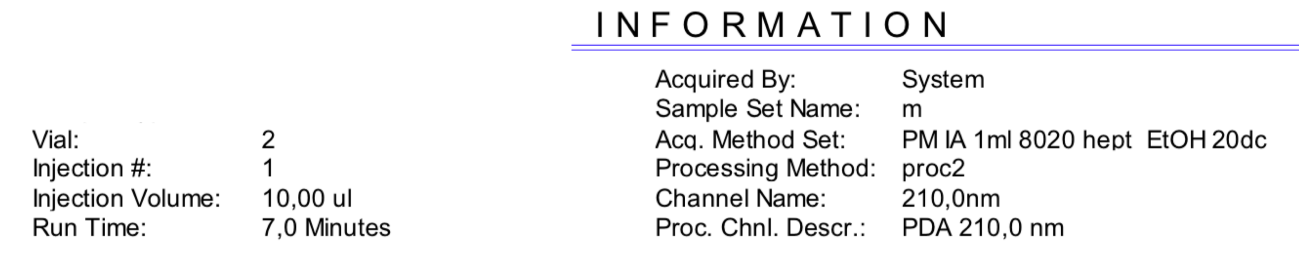
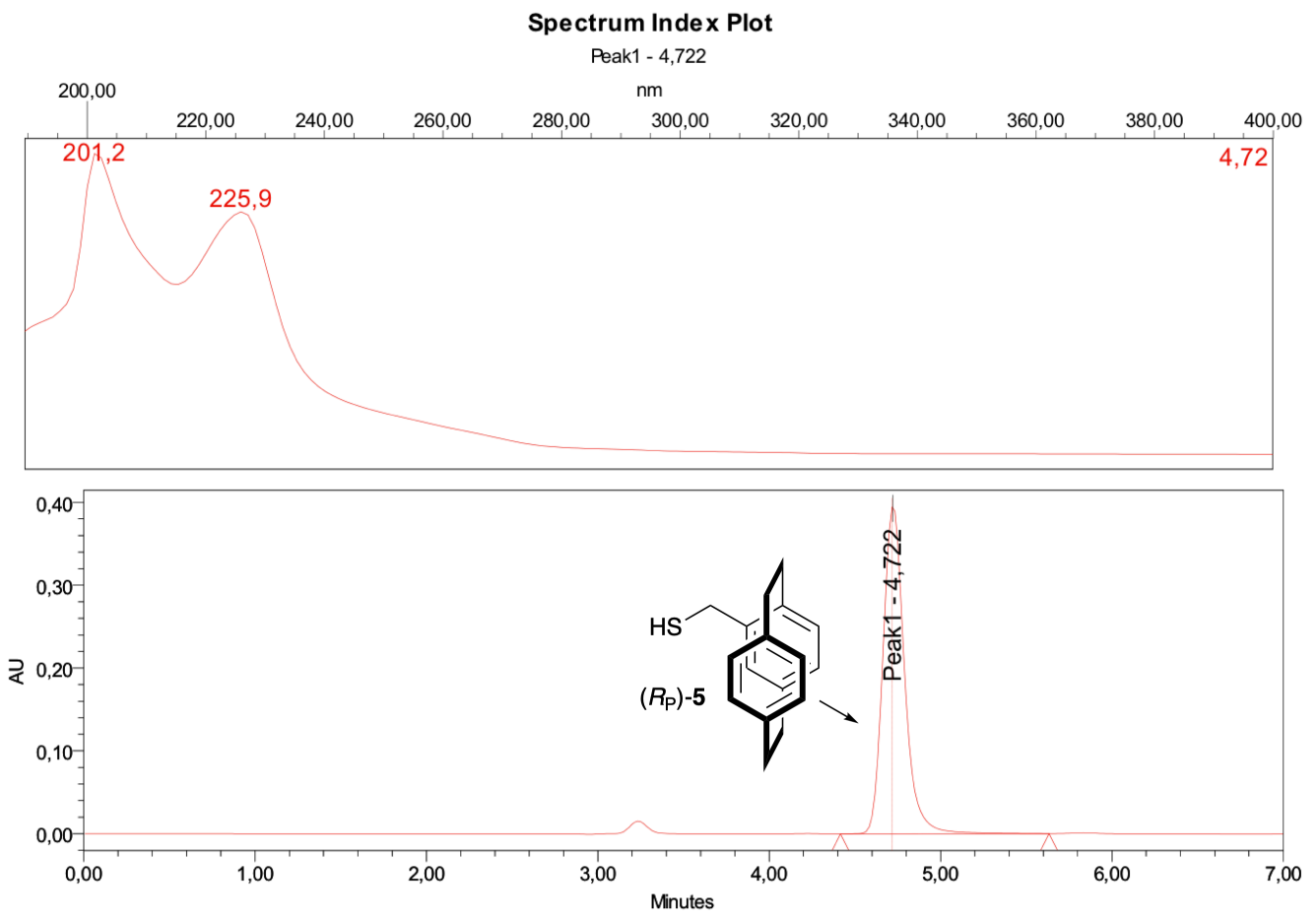

Peak Results

Name RT Area \% Area

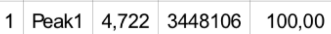




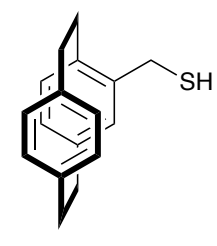

$\left(S_{\mathbf{P}}\right)-5$

$\begin{array}{ll}\text { Vial: } & 2 \\ \text { Injection \#: } & 1 \\ \text { Injection Volume: } & 10,00 \text { ul } \\ \text { Run Time: } & 15,0 \text { Minutes }\end{array}$

I NFORMATION

Acquired By: System

Sample Set Name: I

Acq. Method Set: PM IA $1 \mathrm{ml} 8020$ hept EtOH 20dc

Processing Method: proc

Channel Name: $\quad 210,0 \mathrm{~nm}$

Proc. Chnl. Descr.: PDA 210,0 nm

Spectrum Index Plot

Peak1 - 6,384
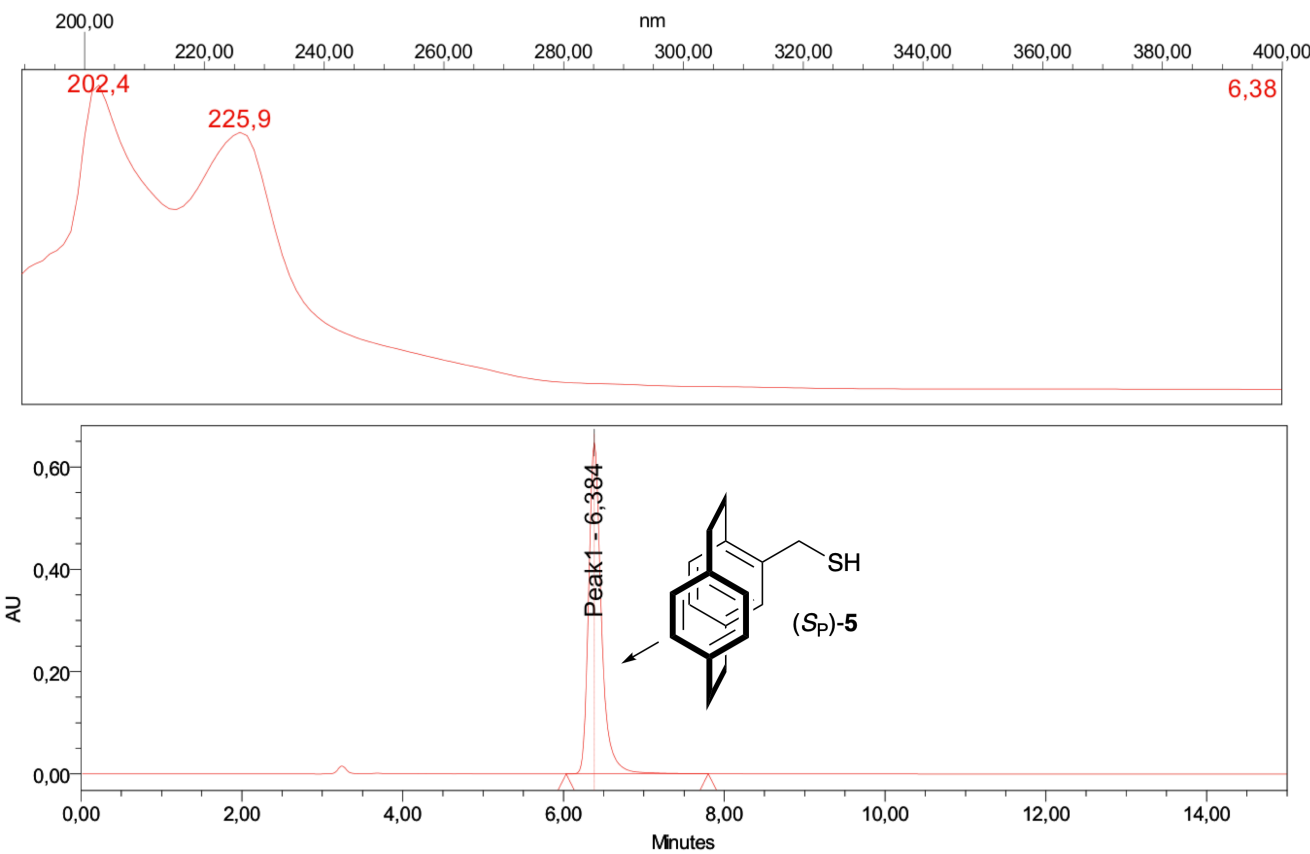

Peak Results

Name RT Area \% Area

1 Peak1 $6,384 \quad 7084777 \quad 100,00$ 
VI - Copies of ${ }^{1} \mathrm{H}$ and ${ }^{13} \mathrm{C}$ Spectra

${ }^{1} \mathrm{H}$ NMR Spectrum of 2a $\left(\mathrm{CDCl}_{3}, 500 \mathrm{MHz}\right)$
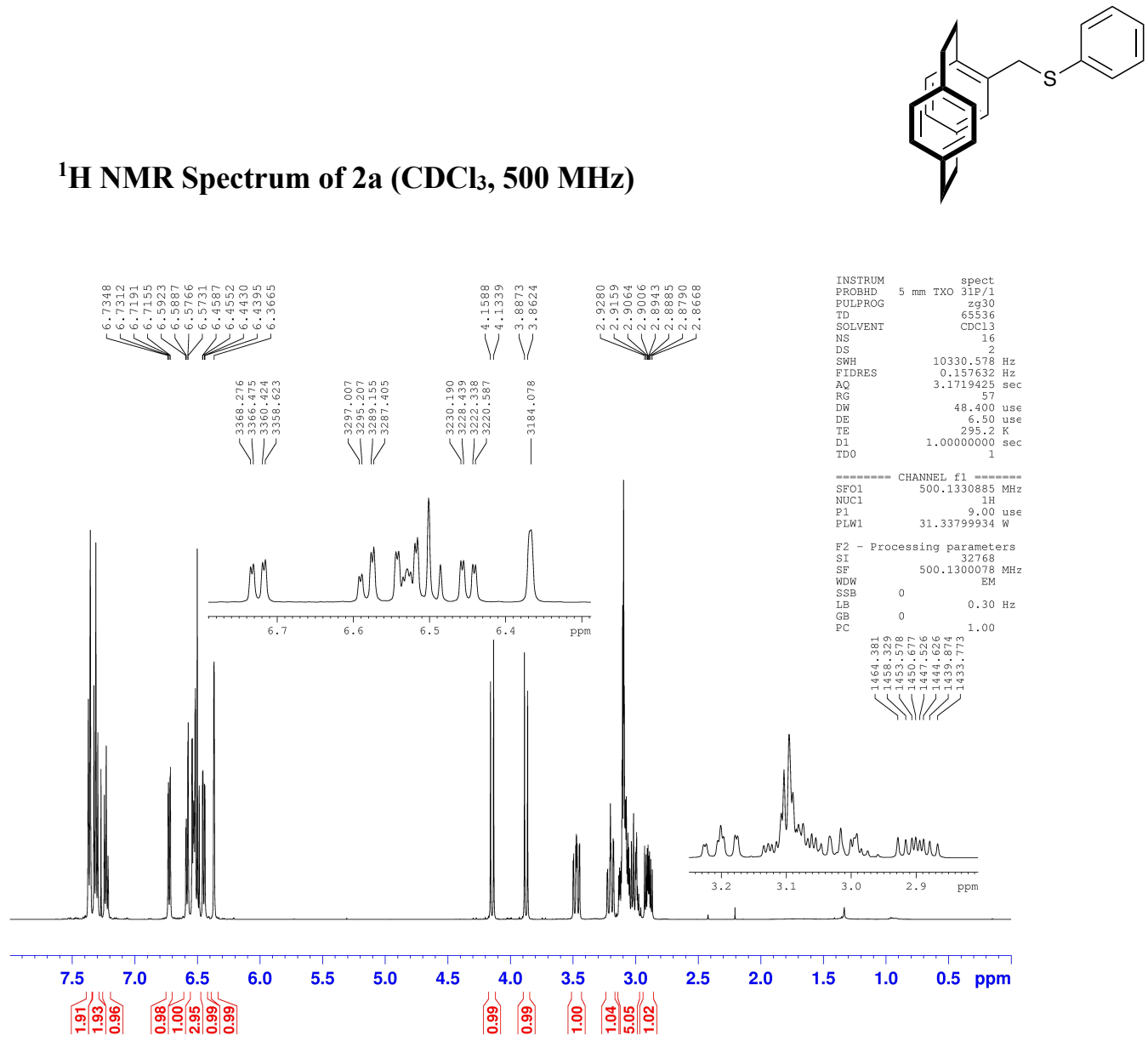

${ }^{13} \mathrm{C}\left\{{ }^{1} \mathrm{H}\right\}$ NMR Spectrum of $2 \mathrm{a}\left(\mathrm{CDCl}_{3}, 101 \mathrm{MHz}\right)$

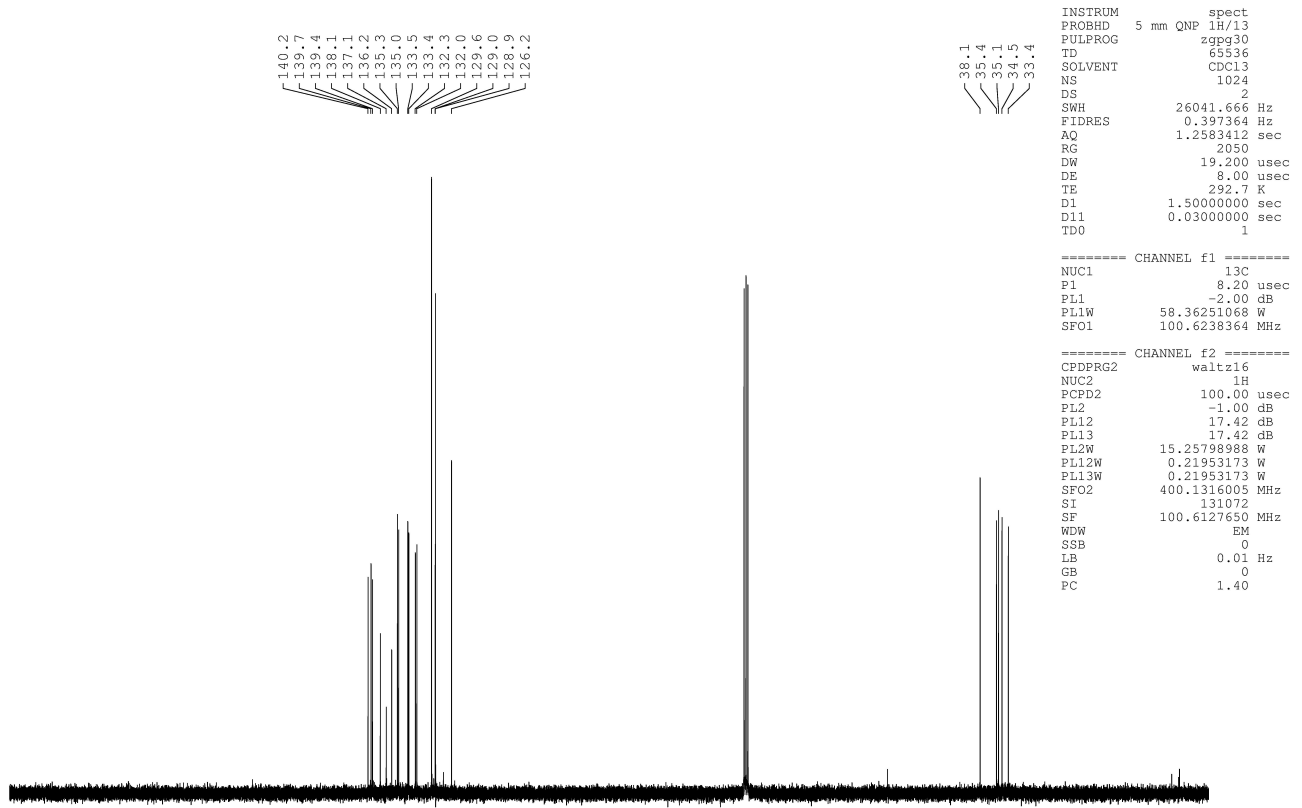

$\begin{array}{llllllllllllllllllll}190 & 180 & 170 & 160 & 150 & 140 & 130 & 120 & 110 & 100 & 90 & 80 & 70 & 60 & 50 & 40 & 30 & 20 & 10 & \text { ppm }\end{array}$ 
${ }^{1} \mathrm{H}$ NMR Spectrum of $2 \mathrm{~b}\left(\mathrm{CDCl}_{3}, 400 \mathrm{MHz}\right)$
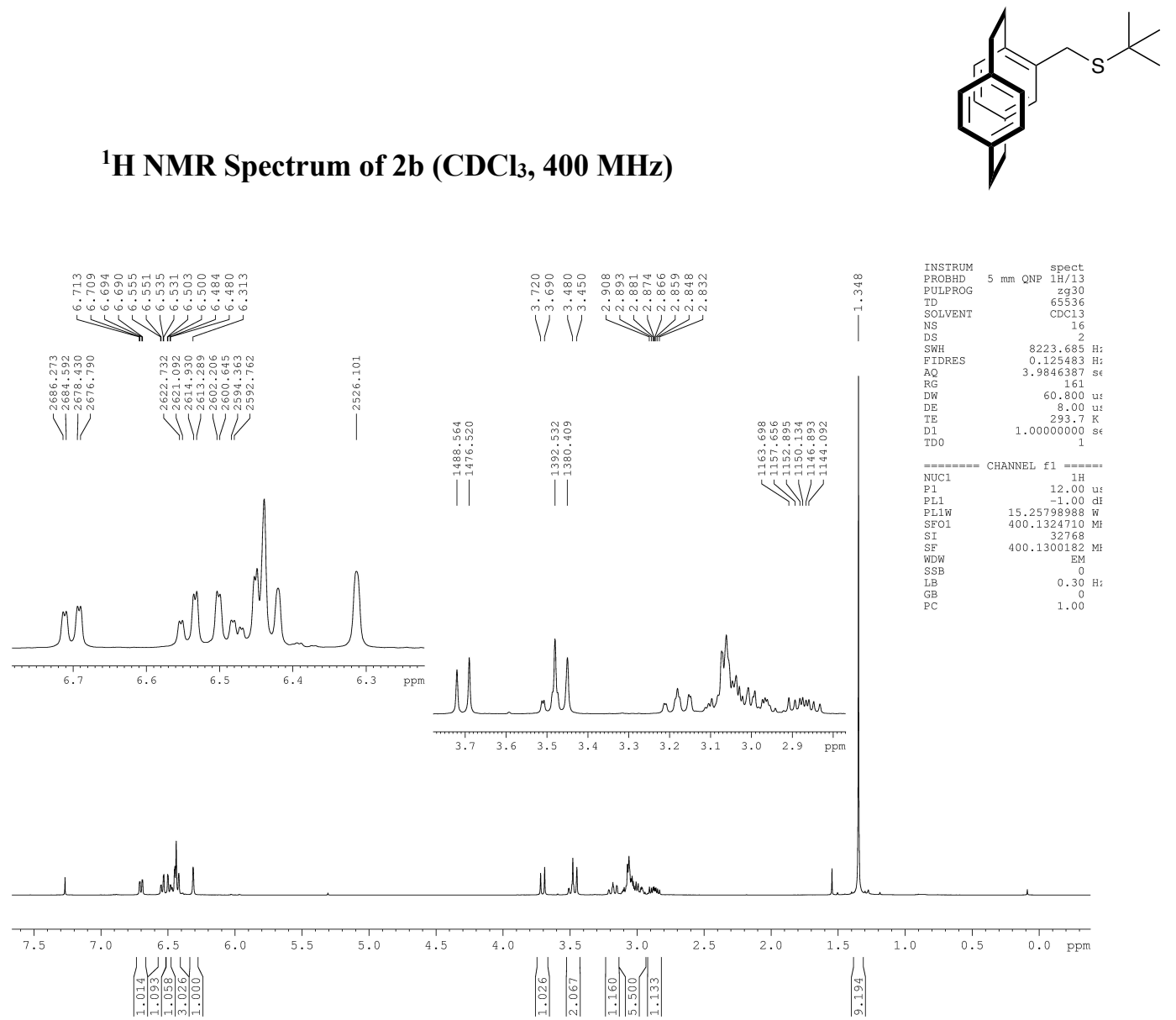

${ }^{13} \mathrm{C}\left\{{ }^{1} \mathrm{H}\right\}$ NMR Spectrum of $2 \mathrm{~b}\left(\mathrm{CDCl}_{3}, 101 \mathrm{MHz}\right)$

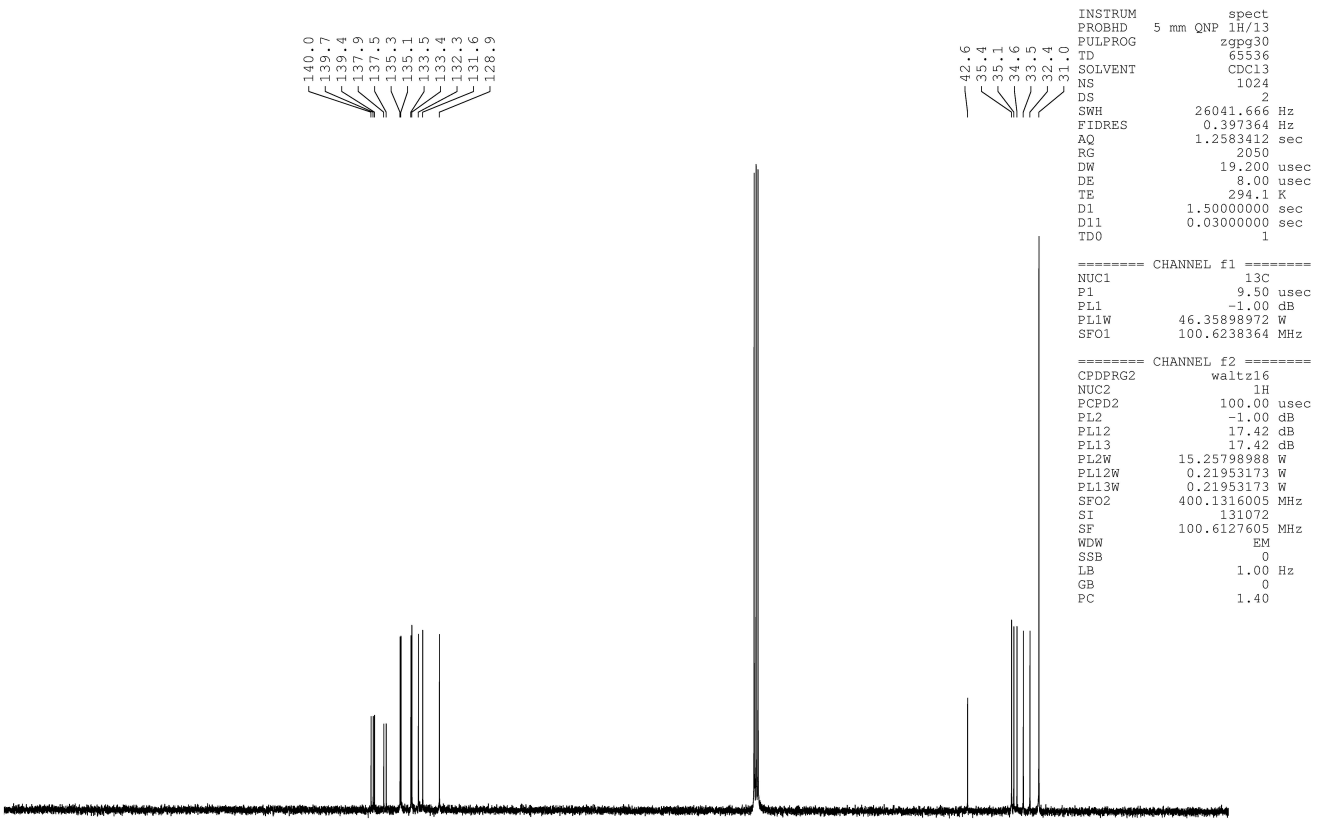

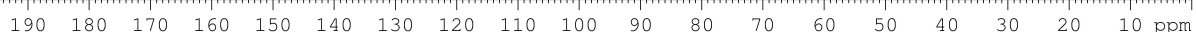


${ }^{1} \mathrm{H}$ NMR Spectrum of $2 \mathrm{c}\left(\mathrm{CDCl}_{3}, 400 \mathrm{MHz}\right)$
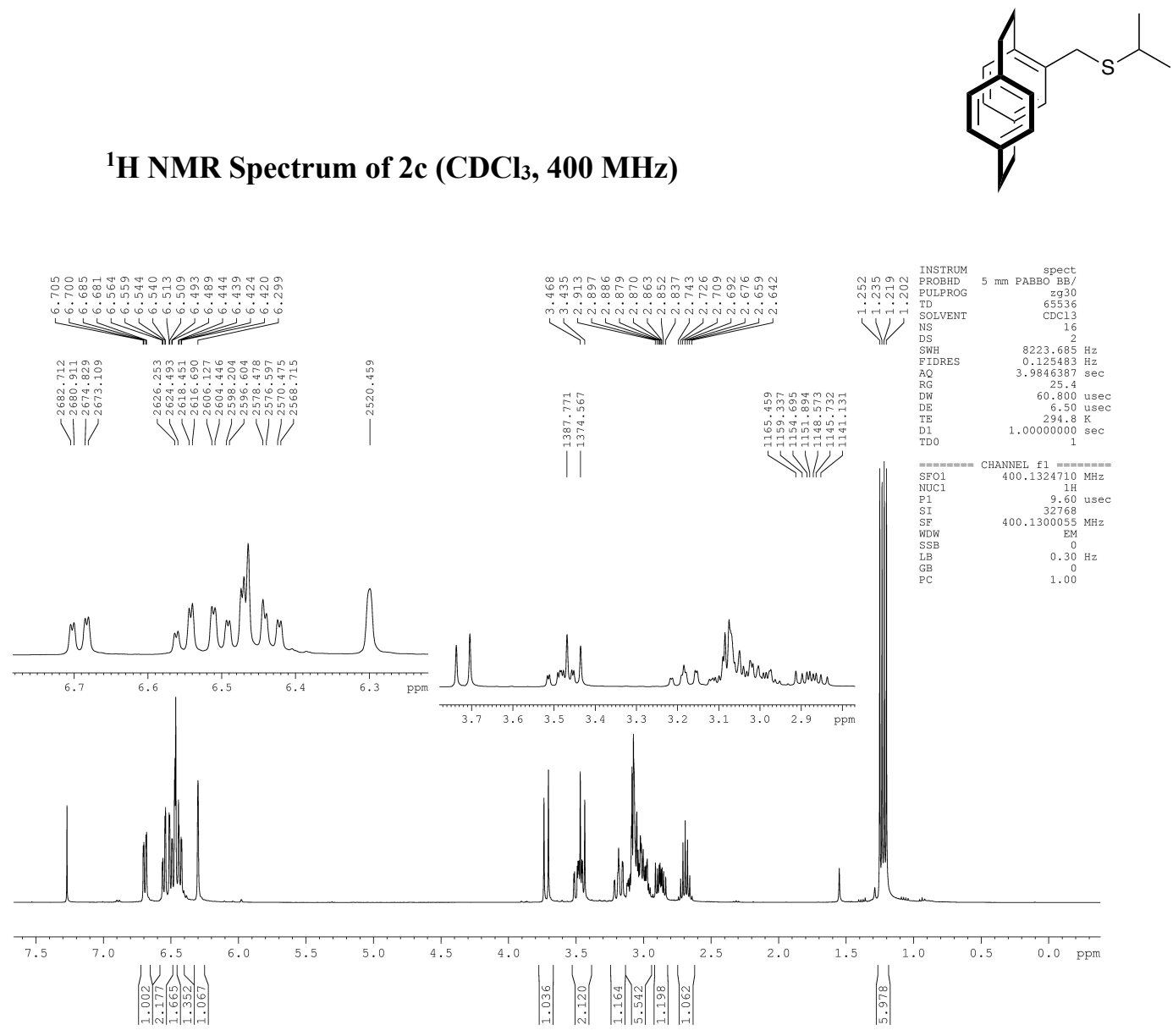

${ }^{13} \mathrm{C}\left\{{ }^{1} \mathrm{H}\right\}$ NMR Spectrum of $2 \mathrm{c}\left(\mathrm{CDCl}_{3}, 101 \mathrm{MHz}\right)$

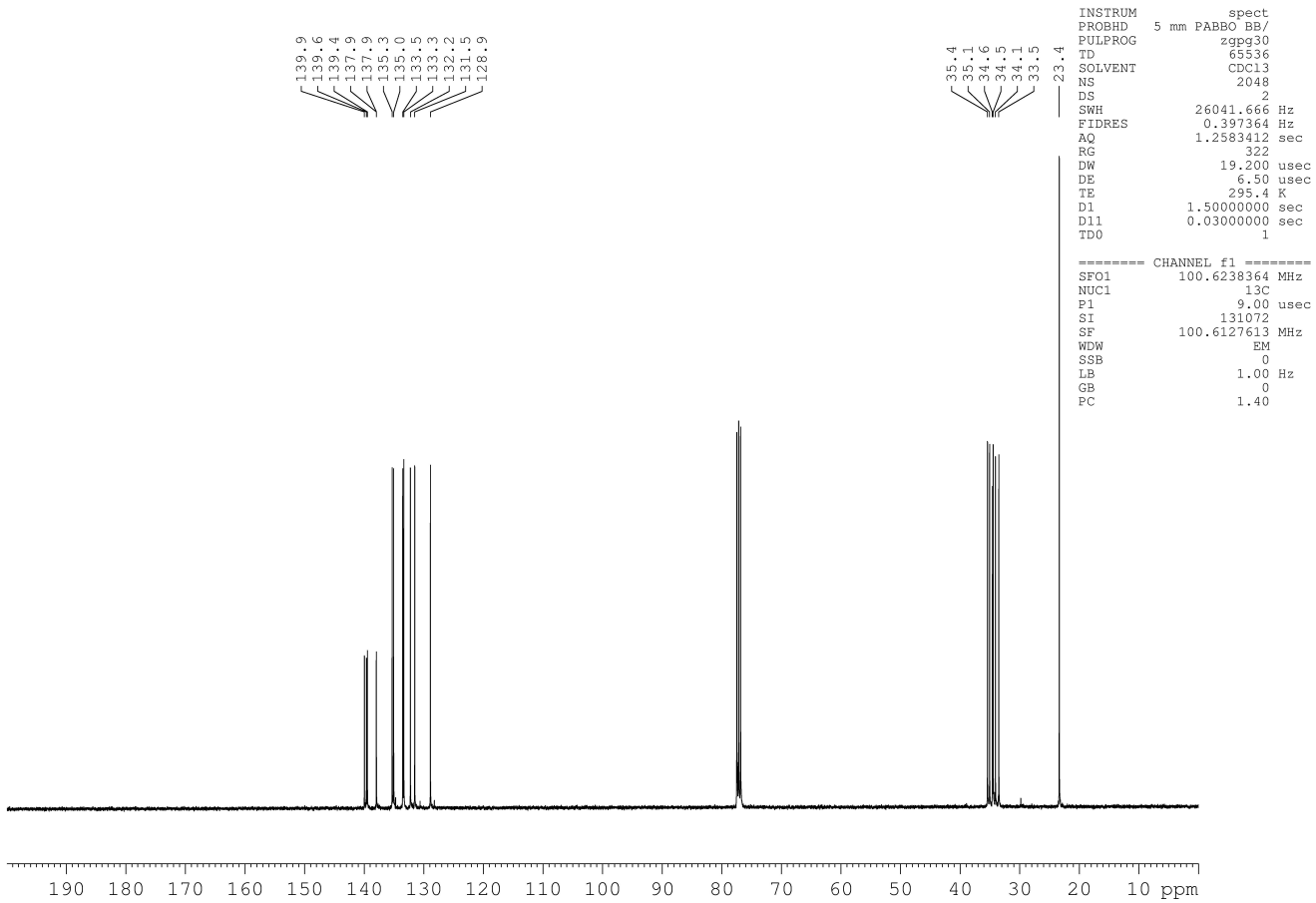


${ }^{1} \mathrm{H}$ NMR Spectrum of $2 \mathrm{~d}\left(\mathrm{CDCl}_{3}, 400 \mathrm{MHz}\right)$
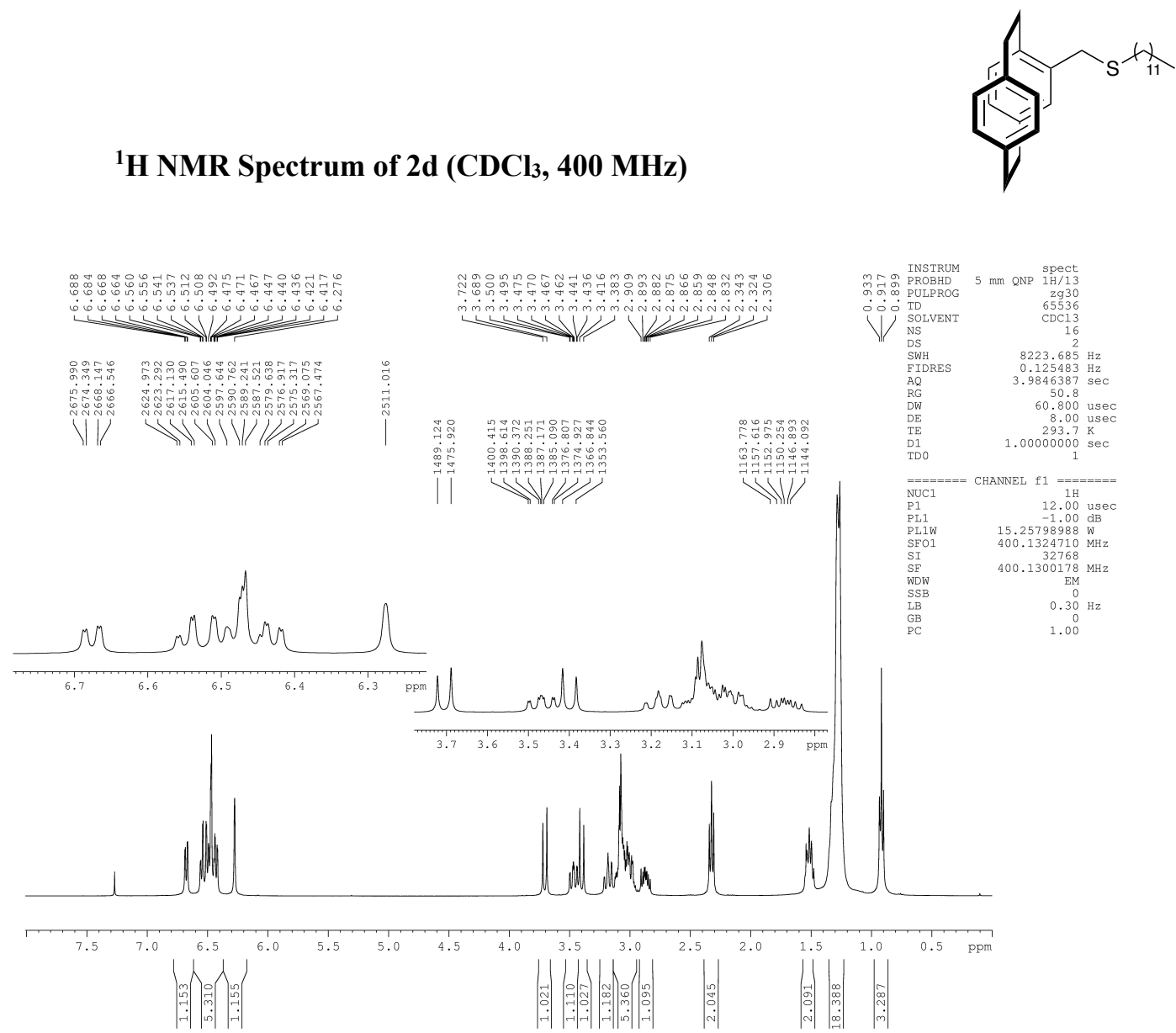

${ }^{13} \mathrm{C}\left\{{ }^{1} \mathrm{H}\right\}$ NMR Spectrum of $2 \mathrm{~d}\left(\mathrm{CDCl}_{3}, 101 \mathrm{MHz}\right)$

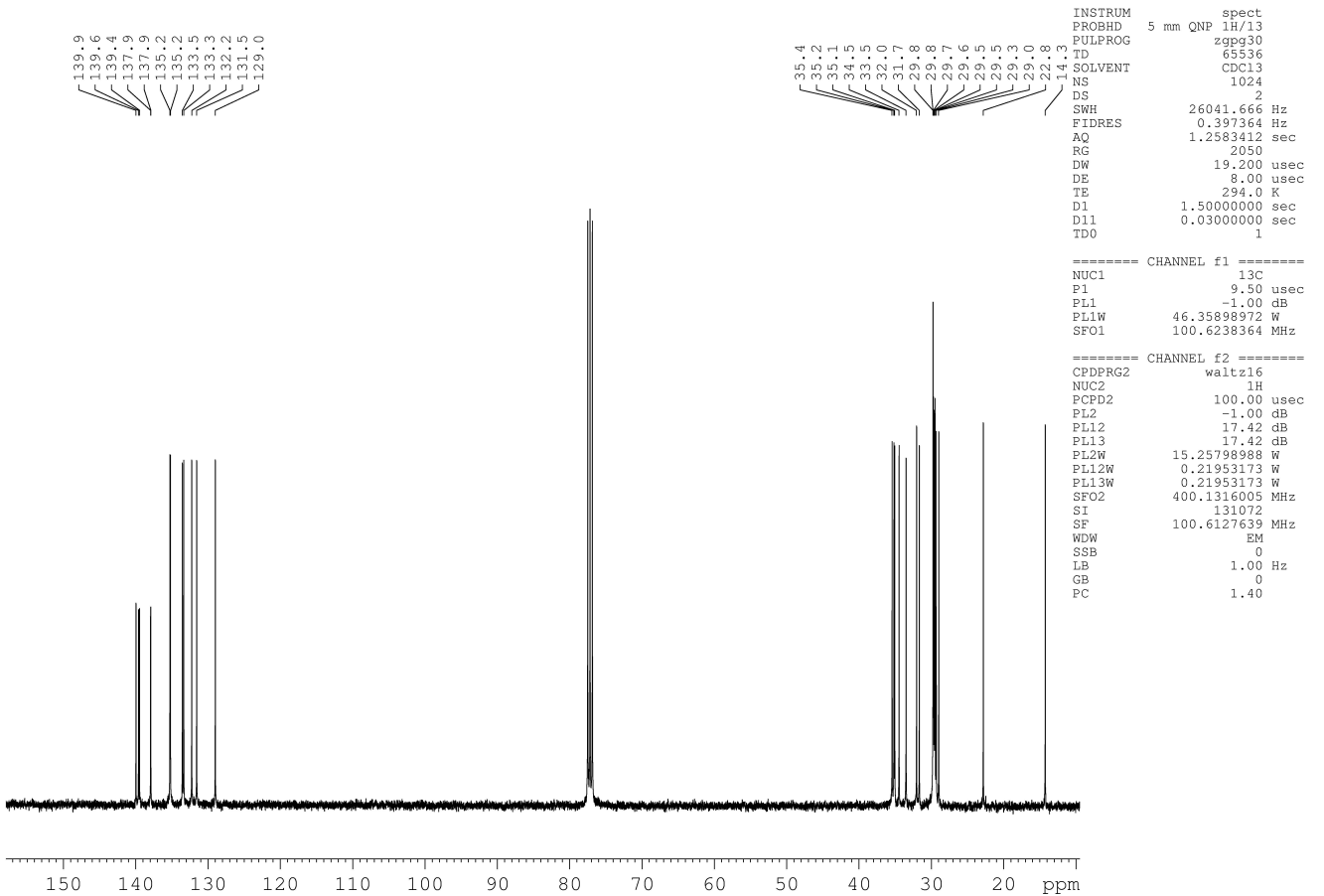


${ }^{1} \mathrm{H}$ NMR Spectrum of $2 \mathrm{e}\left(\mathrm{CDCl}_{3}, 400 \mathrm{MHz}\right)$
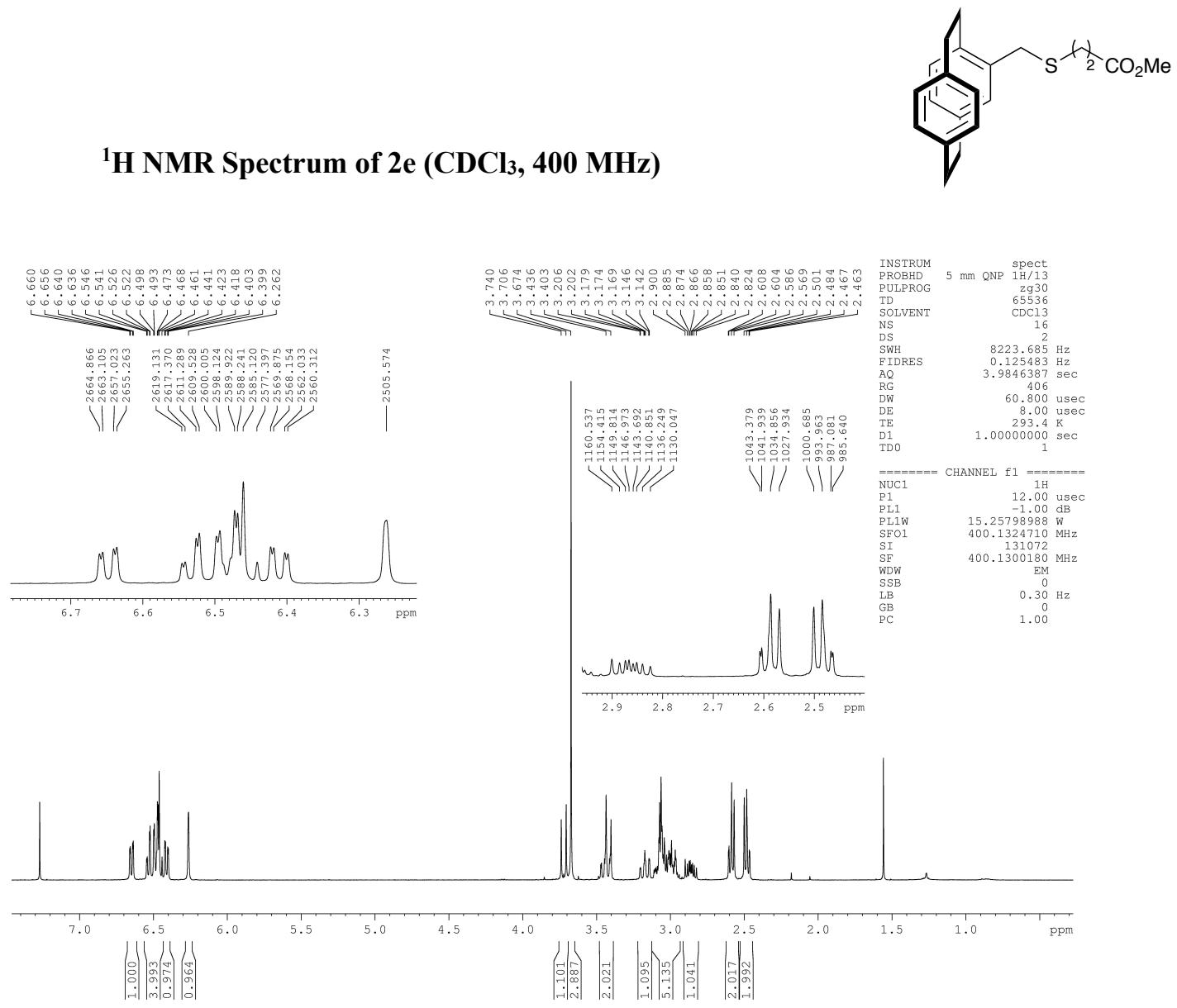

${ }^{13} \mathrm{C}\left\{{ }^{1} \mathrm{H}\right\}$ NMR Spectrum of $2 \mathrm{e}\left(\mathrm{CDCl}_{3}, 101 \mathrm{MHz}\right)$

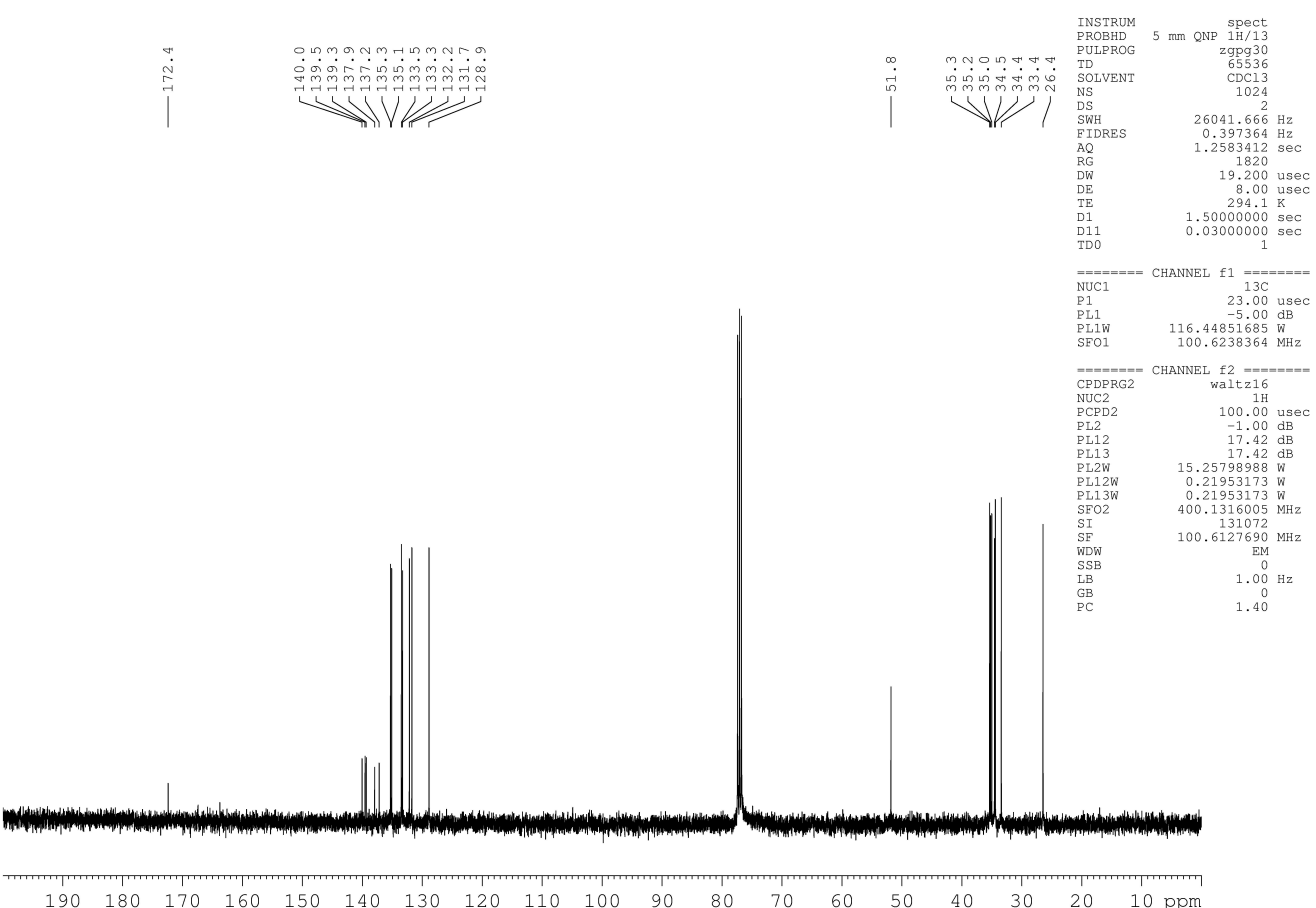


${ }^{1} \mathrm{H}$ NMR Spectrum of $2 \mathrm{f}\left(\mathrm{CDCl}_{3}, 400 \mathrm{MHz}\right)$
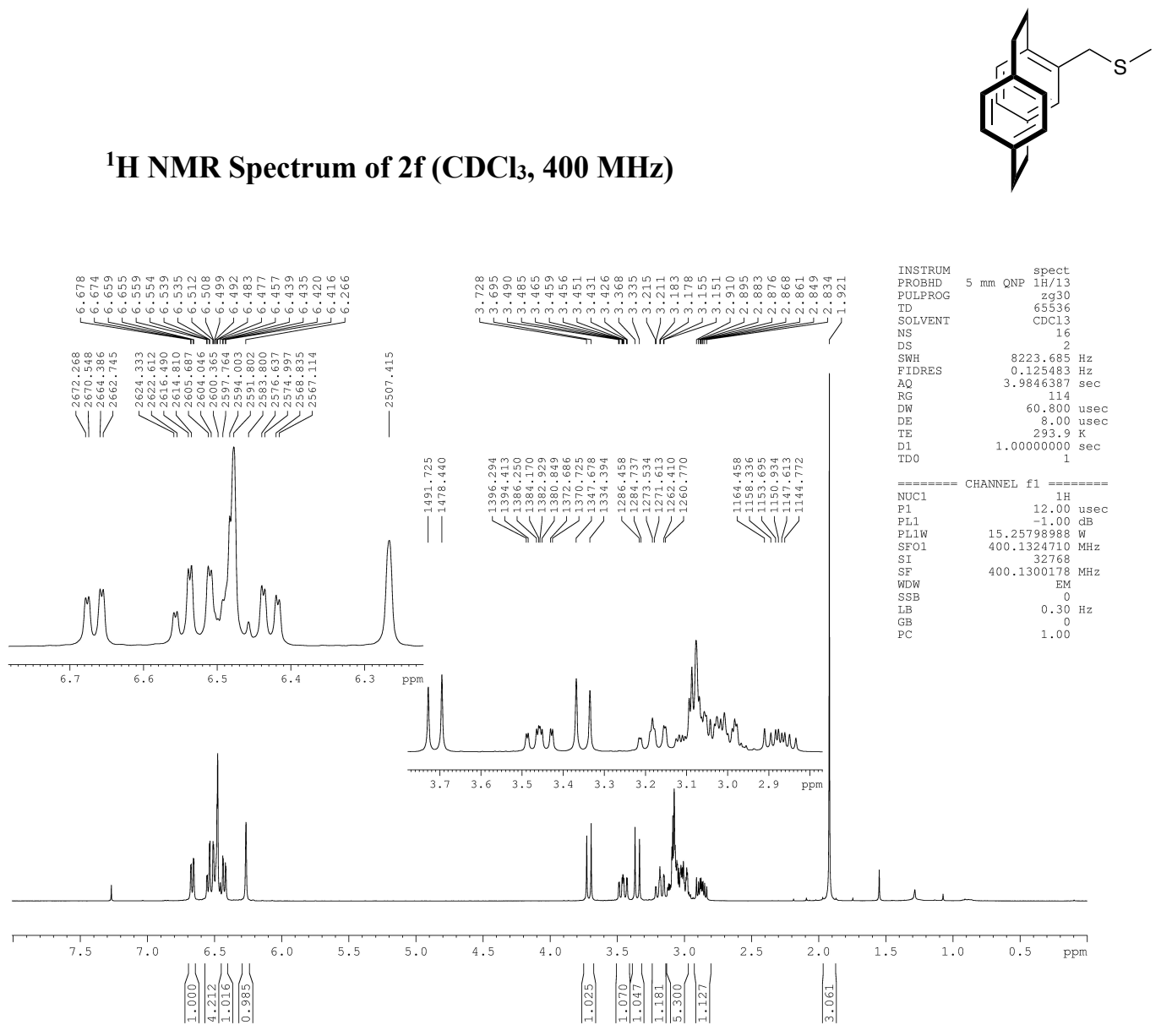

${ }^{13} \mathrm{C}\left\{{ }^{1} \mathrm{H}\right\}$ NMR Spectrum of $2 \mathrm{f}\left(\mathrm{CDCl}_{3}, 101 \mathrm{MHz}\right)$

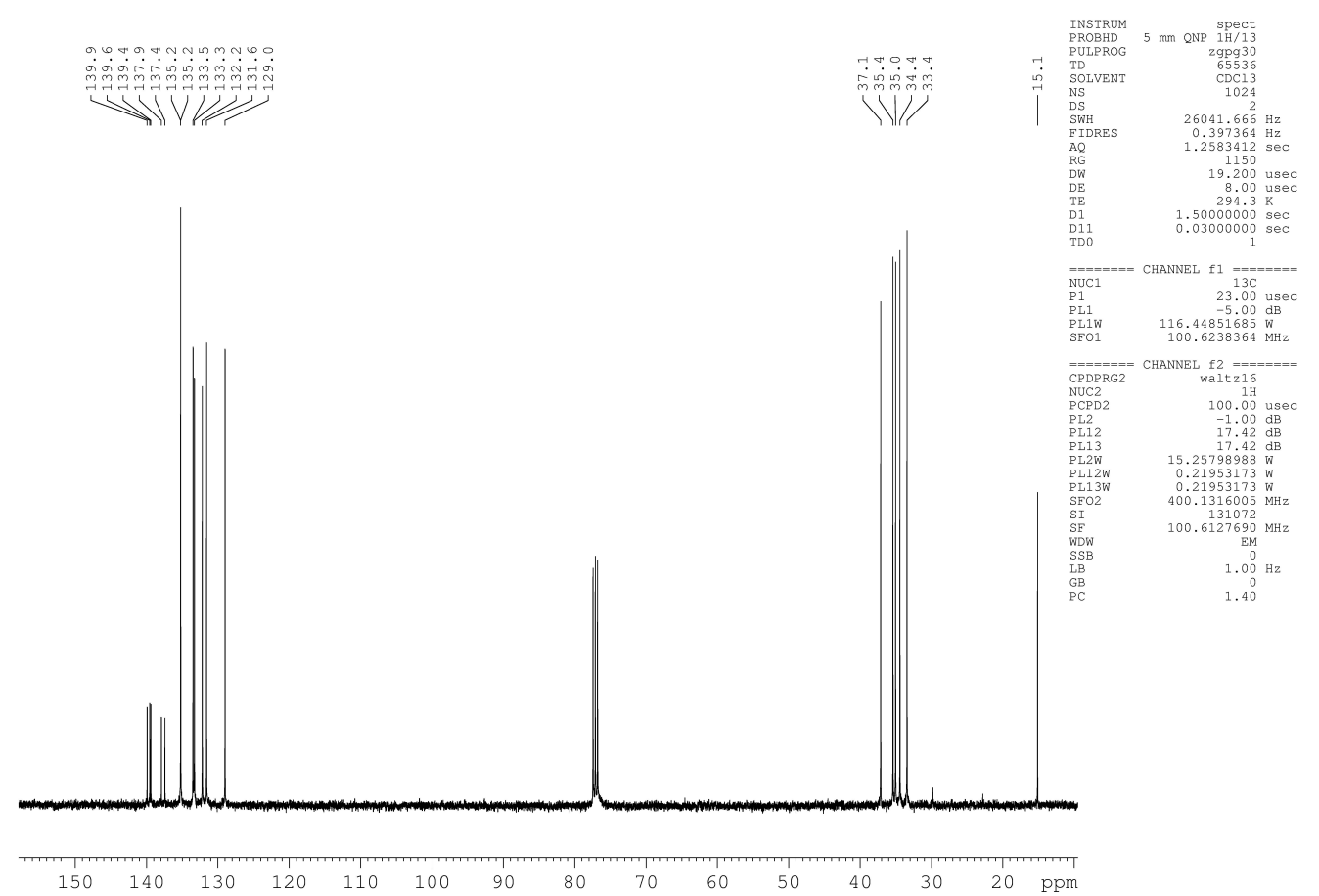


${ }^{1} \mathrm{H}$ NMR Spectrum of $\left(S_{\mathrm{P}}, R\right) *-4 a\left(\mathrm{CDCl}_{3}, 400 \mathrm{MHz}\right)$
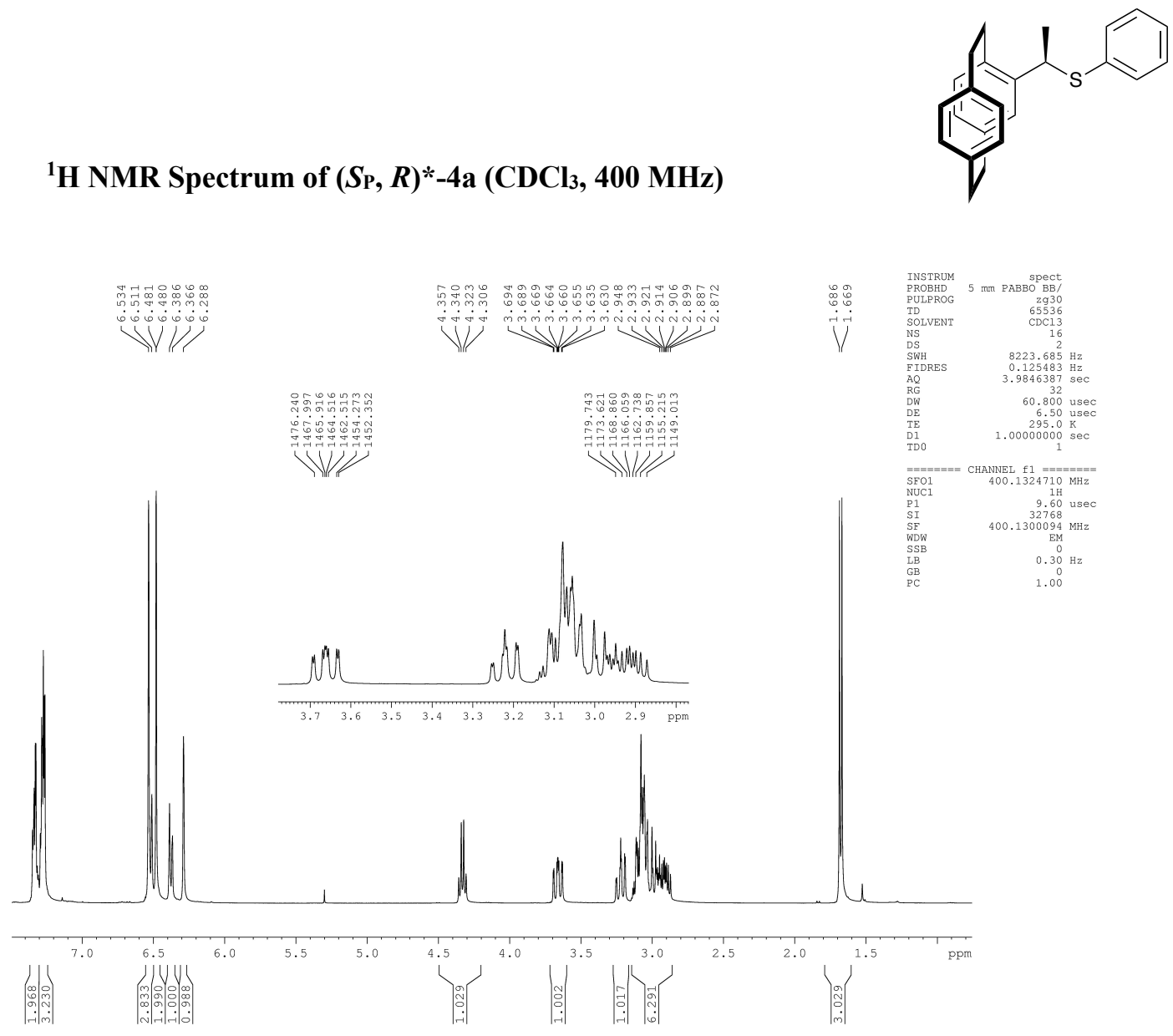

${ }^{13} \mathrm{C}\left\{{ }^{1} \mathrm{H}\right\}$ NMR Spectrum of $\left(S_{\mathrm{P}}, R\right) *-4 a\left(\mathrm{CDCl}_{3}, 101 \mathrm{MHz}\right)$

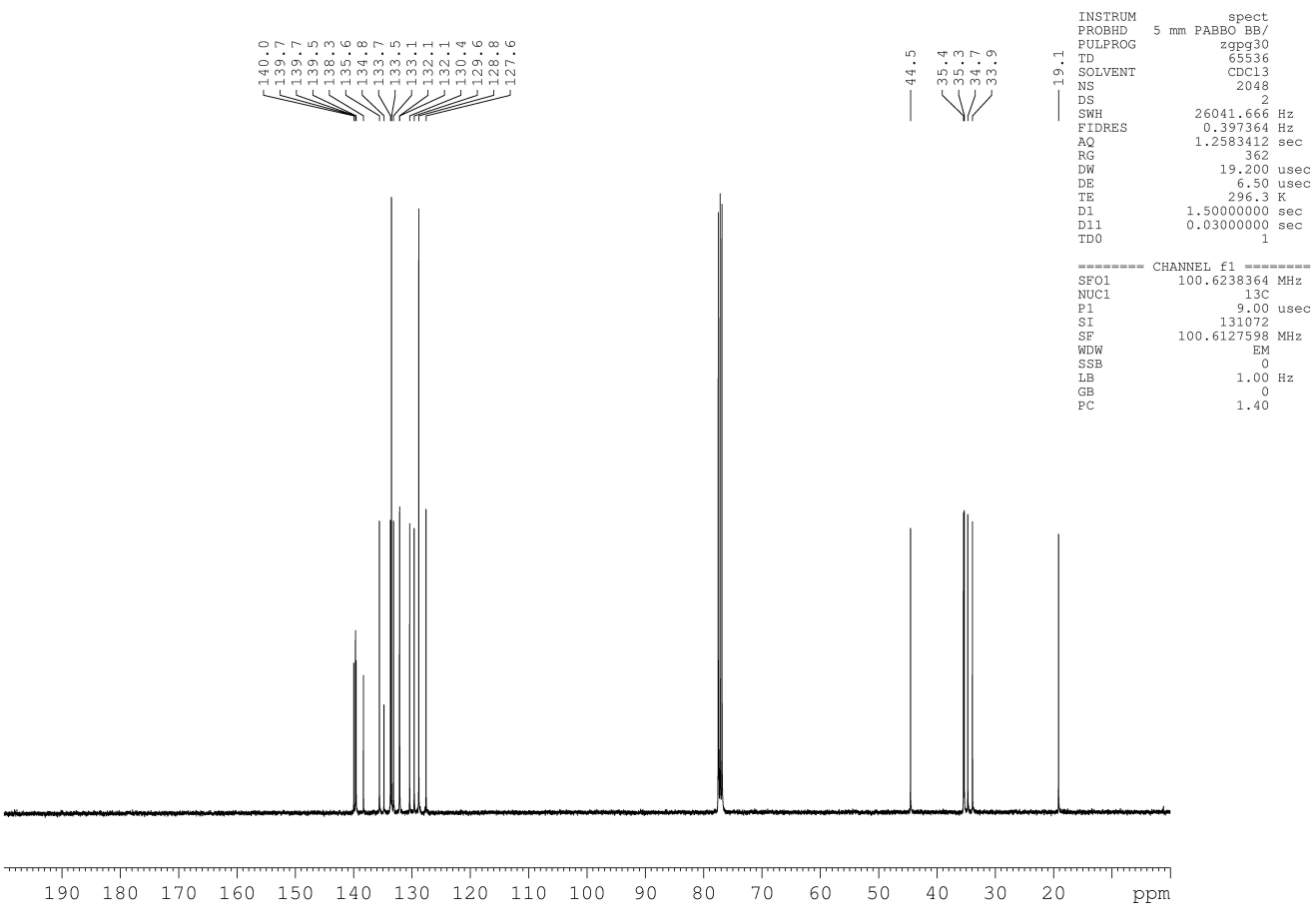


${ }^{1} \mathrm{H}$ NMR Spectrum of $\left(S_{\mathrm{P}}, \mathrm{S}\right) *-4 \mathrm{a}\left(\mathrm{CDCl}_{3}, 400 \mathrm{MHz}\right)$
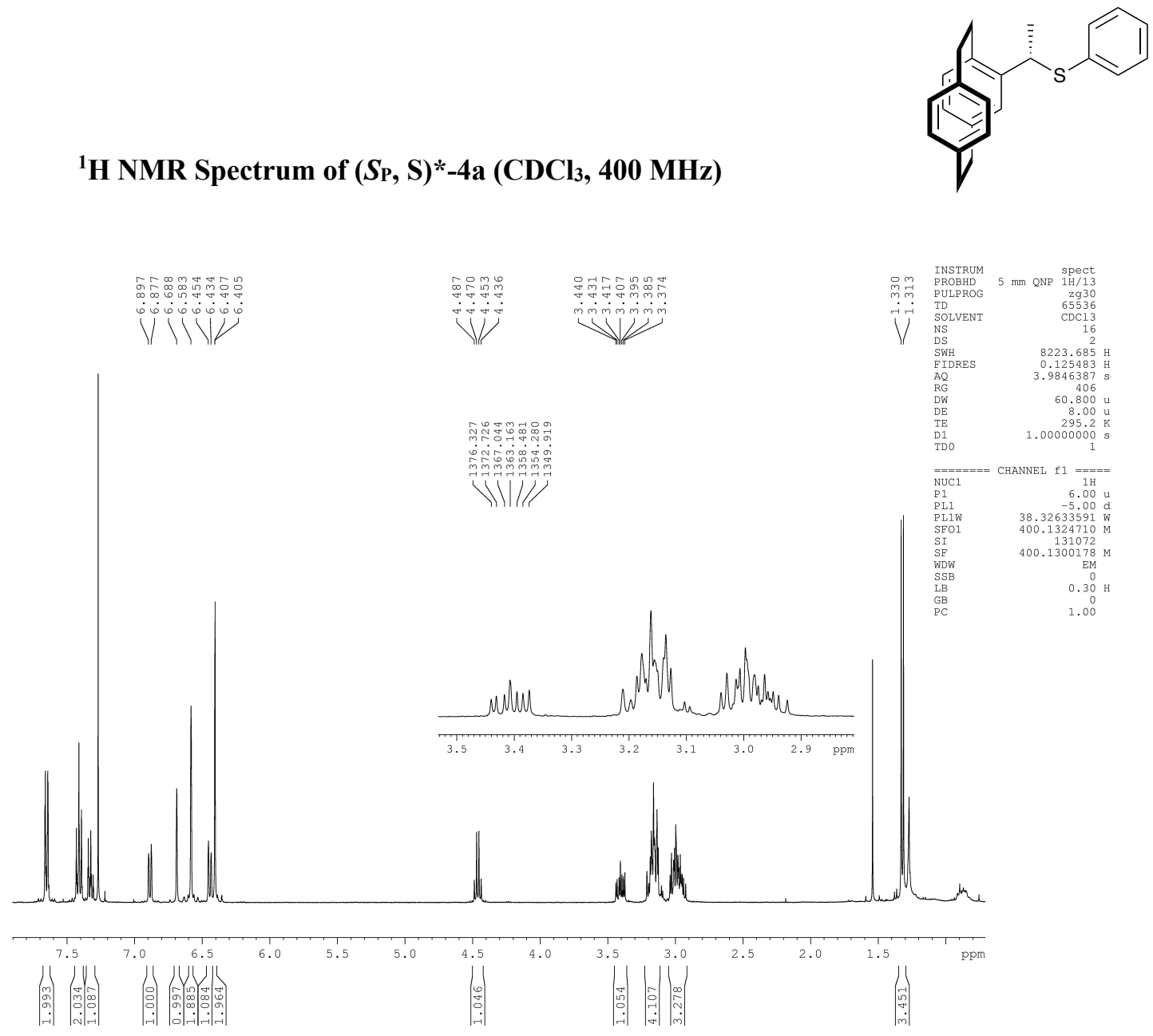

${ }^{13} \mathrm{C}\left\{{ }^{1} \mathrm{H}\right\}$ NMR Spectrum of $\left(S_{\mathrm{P}}, S\right) *-4 a\left(\mathrm{CDCl}_{3}, 101 \mathrm{MHz}\right)$

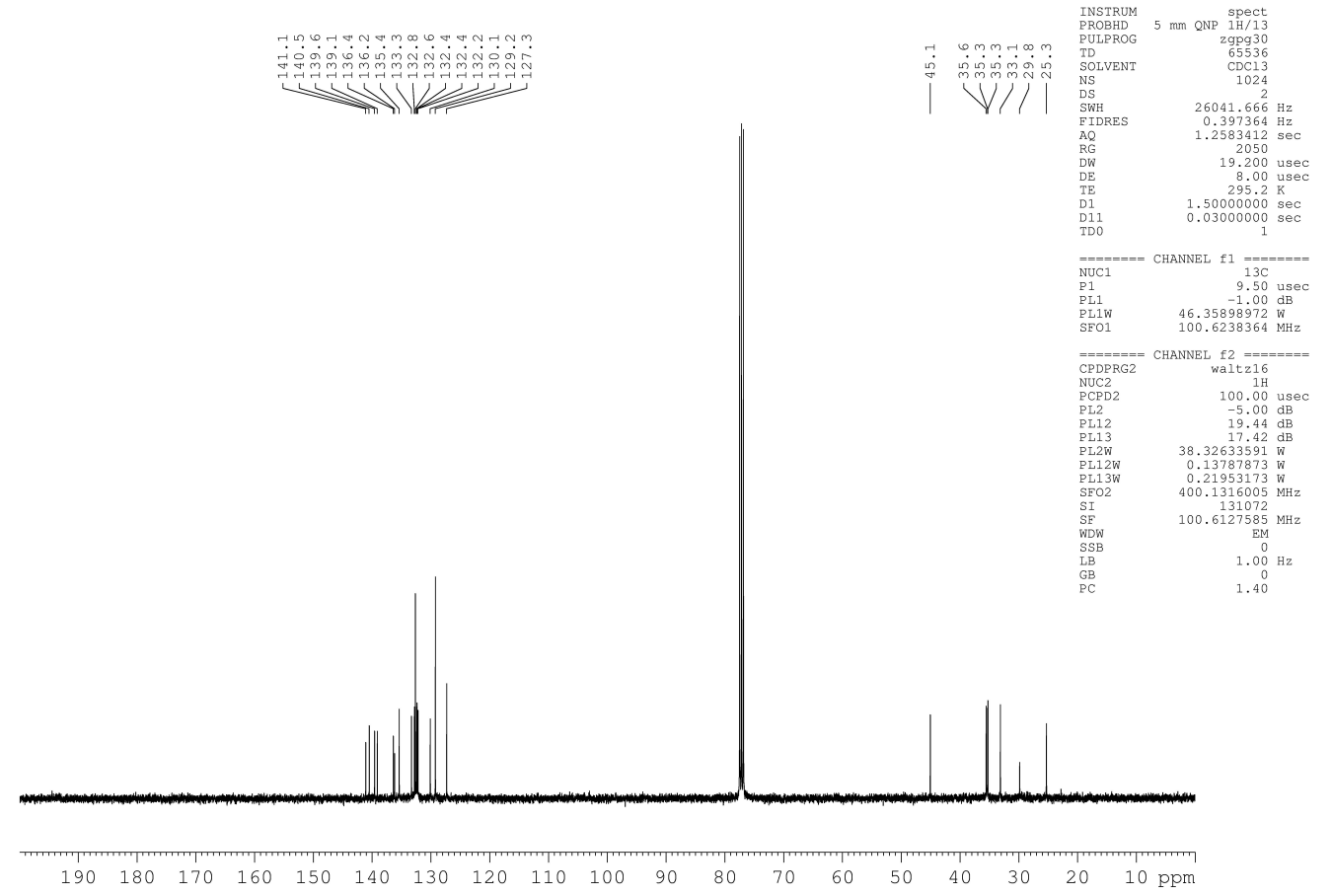


${ }^{1} \mathrm{H}$ NMR Spectrum of $\left(S_{\mathrm{P}}, \mathrm{R}\right) *-4 \mathrm{~b}\left(\mathrm{CDCl}_{3}, 400 \mathrm{MHz}\right)$
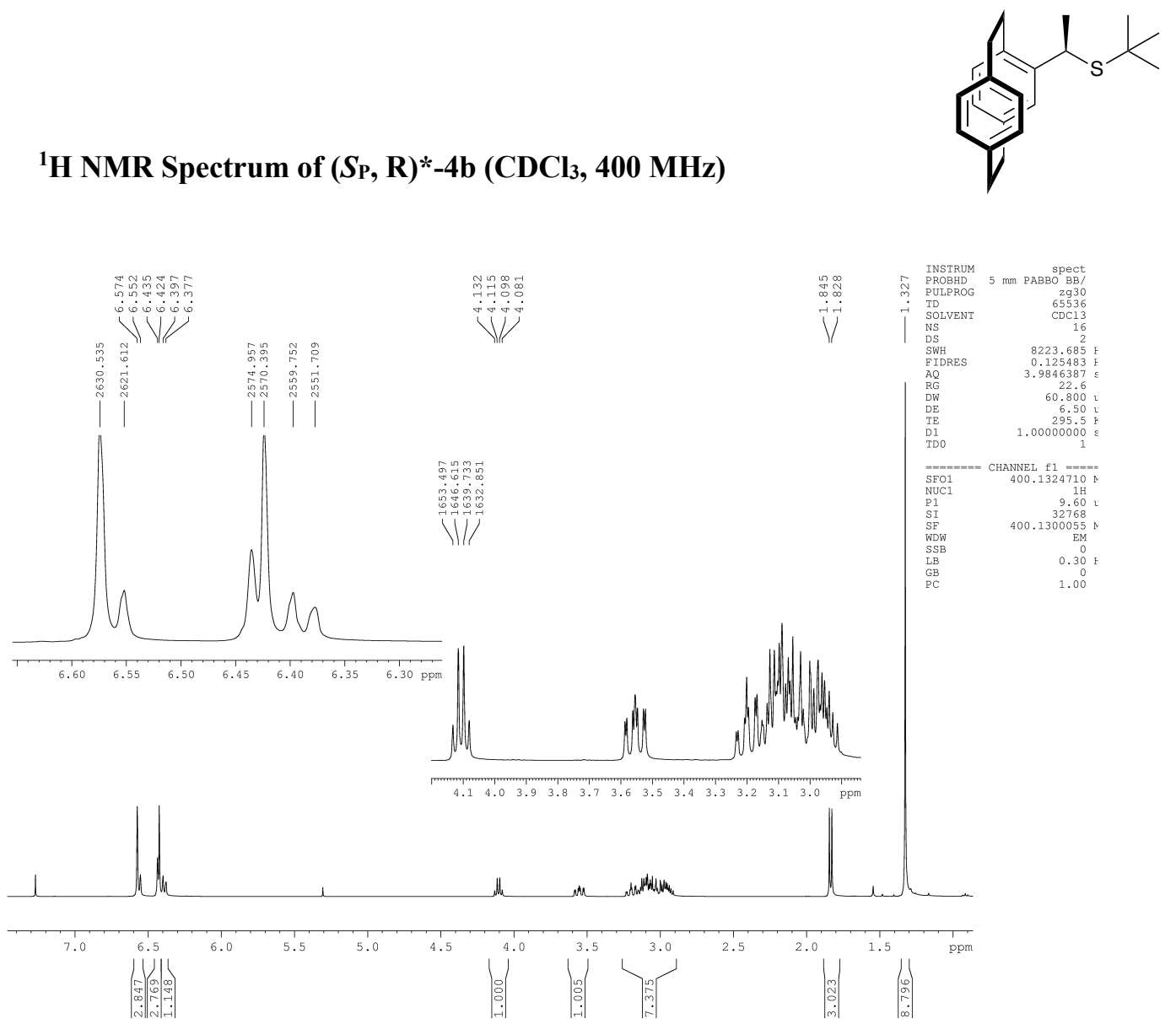

${ }^{13} \mathrm{C}\left\{{ }^{1} \mathrm{H}\right\}$ NMR Spectrum of $\left(S_{\mathrm{P}}, R\right){ }^{*}-4 \mathrm{~b}\left(\mathrm{CDCl}_{3}, 101 \mathrm{MHz}\right)$

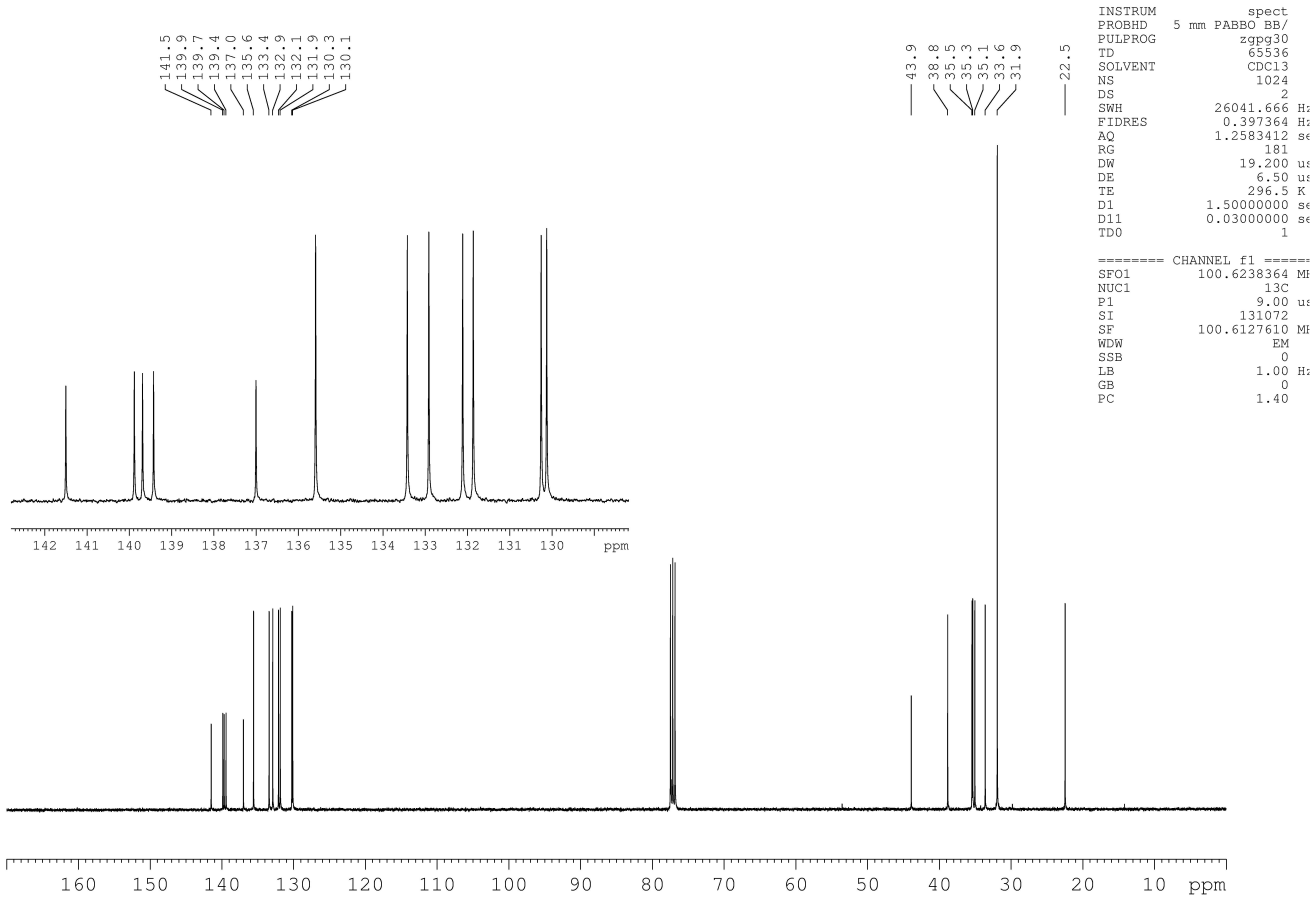


${ }^{1} \mathrm{H}$ NMR Spectrum of $\left(S_{\mathrm{P}}, \mathrm{S}\right) *-4 \mathrm{~b}\left(\mathrm{CDCl}_{3}, 400 \mathrm{MHz}\right)$
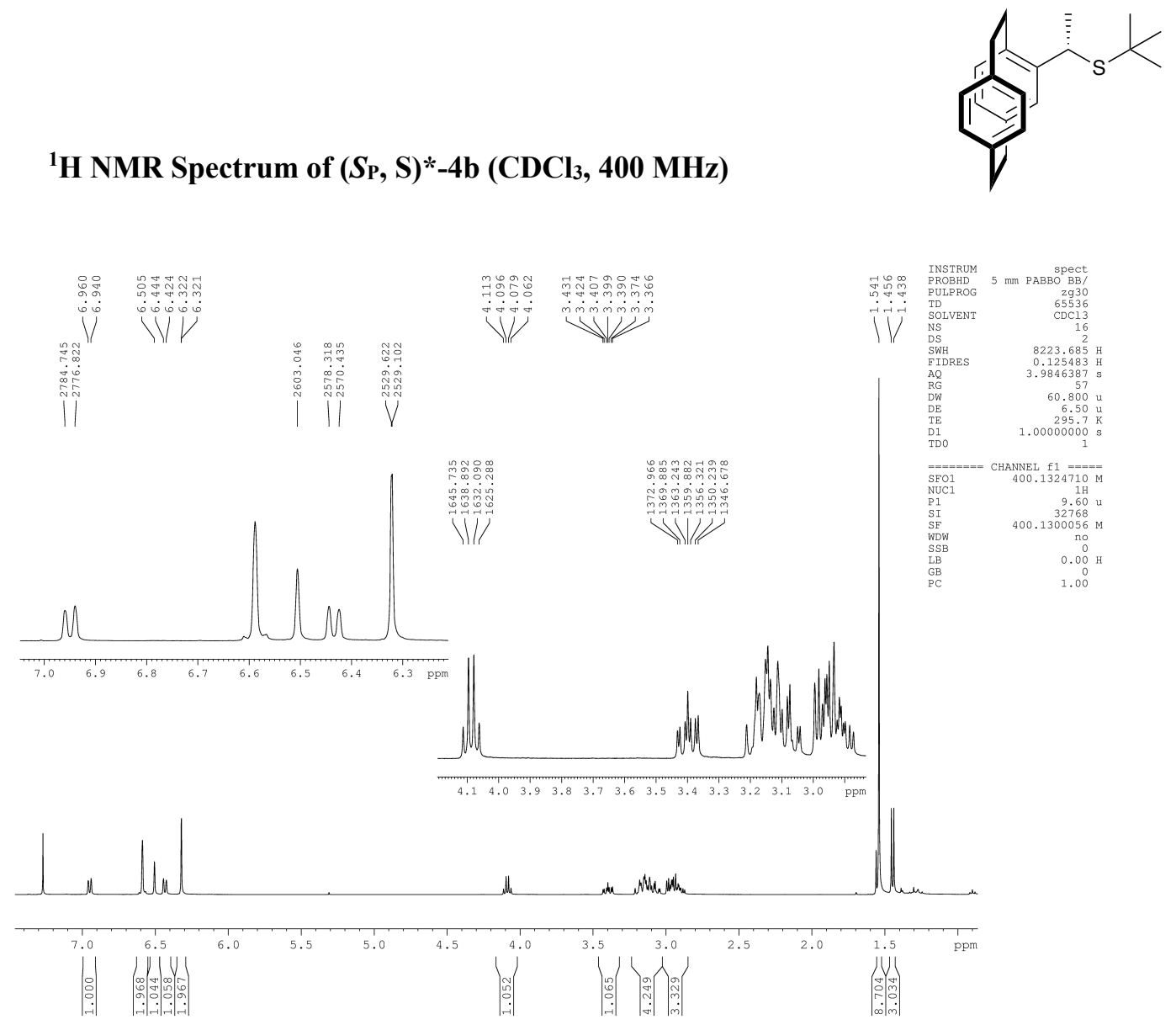

${ }^{13} \mathrm{C}\left\{{ }^{1} \mathrm{H}\right\}$ NMR Spectrum of $\left(S_{\mathrm{P}}, S\right) *-4 \mathrm{~b}\left(\mathrm{CDCl}_{3}, 101 \mathrm{MHz}\right)$

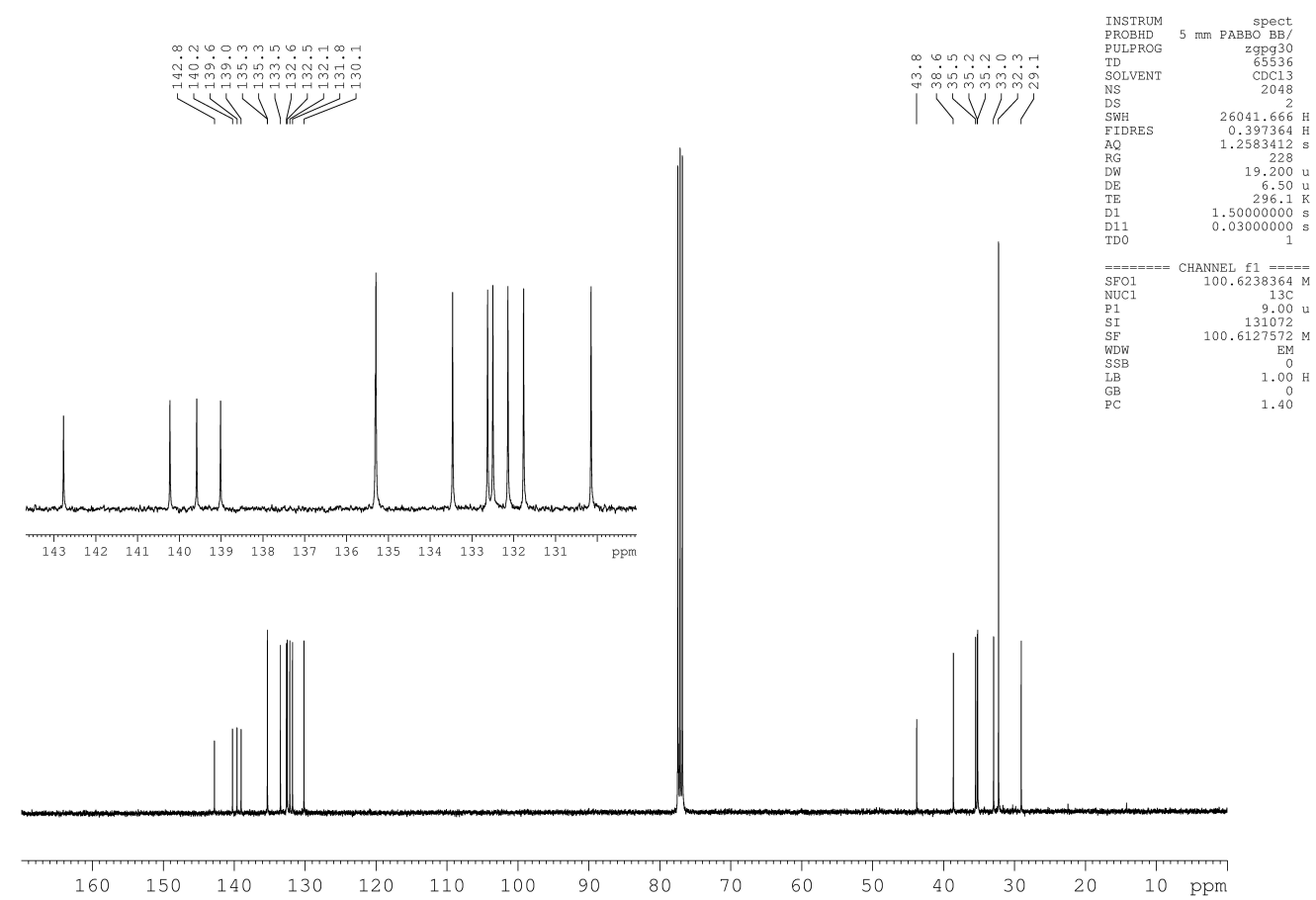


${ }^{1} \mathrm{H}$ NMR Spectrum of $\left(S_{\mathrm{P}}, \mathrm{R}\right) *-4 \mathrm{c}\left(\mathrm{CDCl}_{3}, 400 \mathrm{MHz}\right)$
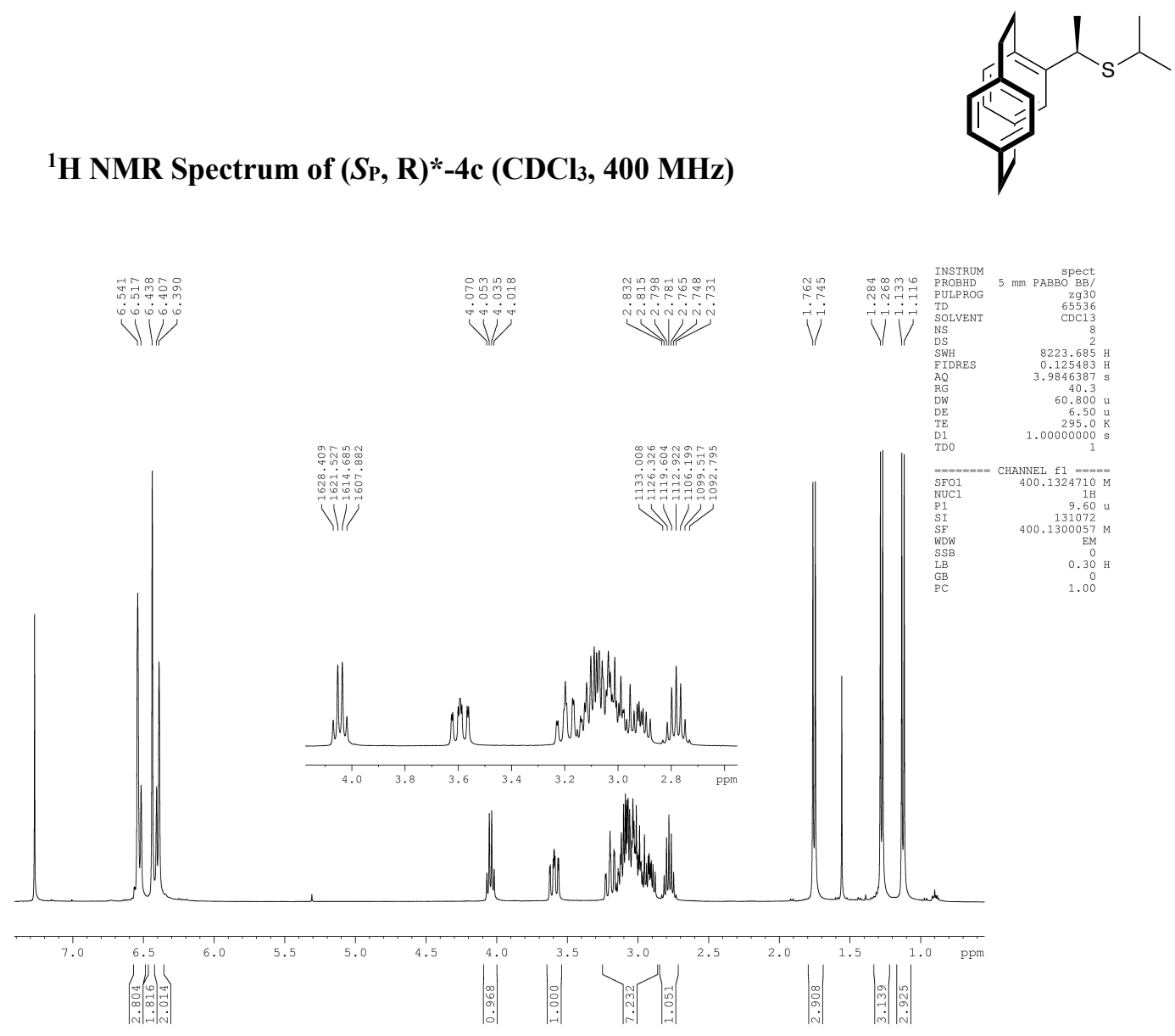

${ }^{13} \mathrm{C}\left\{{ }^{1} \mathrm{H}\right\}$ NMR Spectrum of $\left(S_{\mathrm{P}}, R\right) *-4 \mathrm{c}\left(\mathrm{CDCl}_{3}, 101 \mathrm{MHz}\right)$

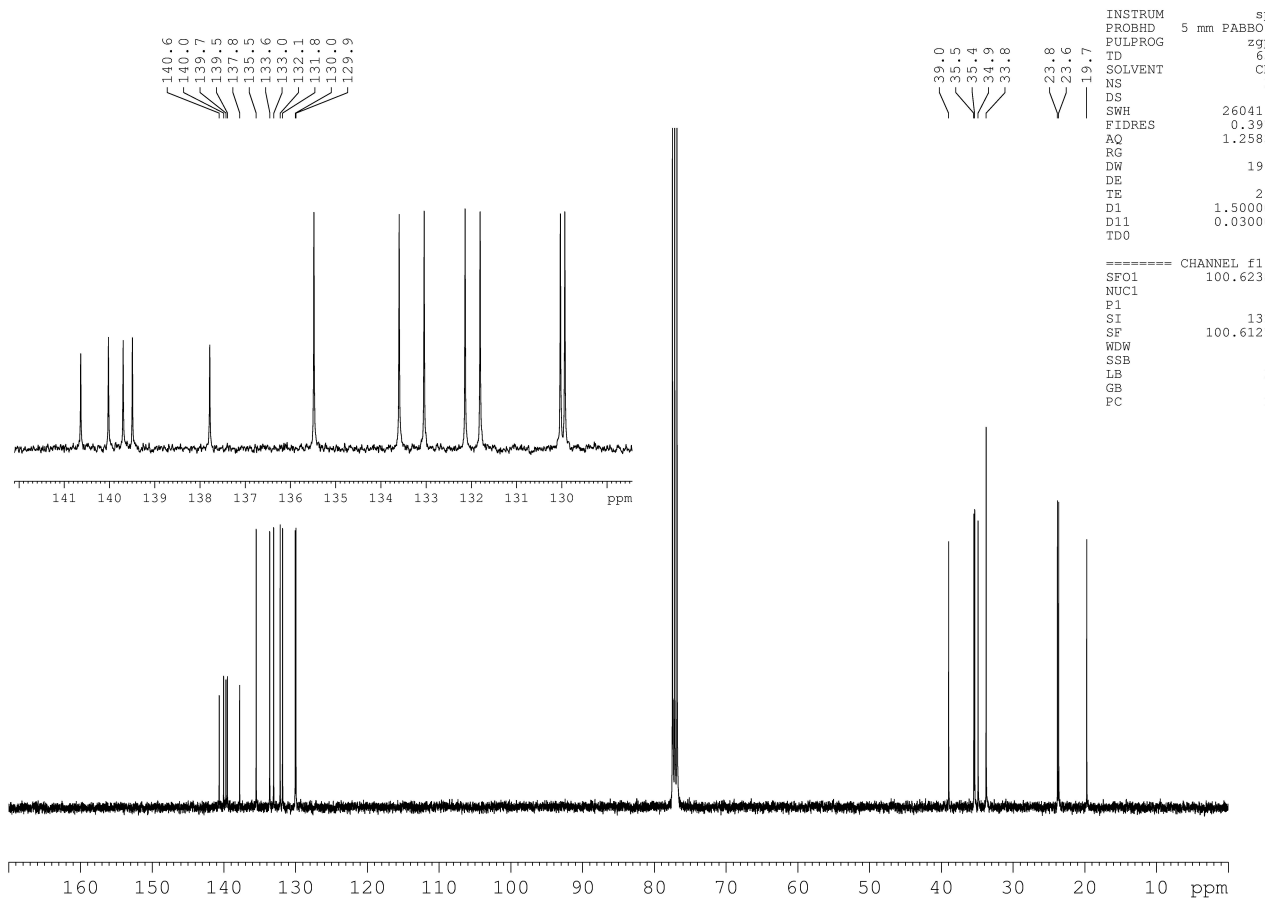


${ }^{1} \mathrm{H}$ NMR Spectrum of $\left(S_{\mathrm{P}}, \mathrm{S}\right) *-4 \mathrm{c}\left(\mathrm{CDCl}_{3}, 400 \mathrm{MHz}\right)$
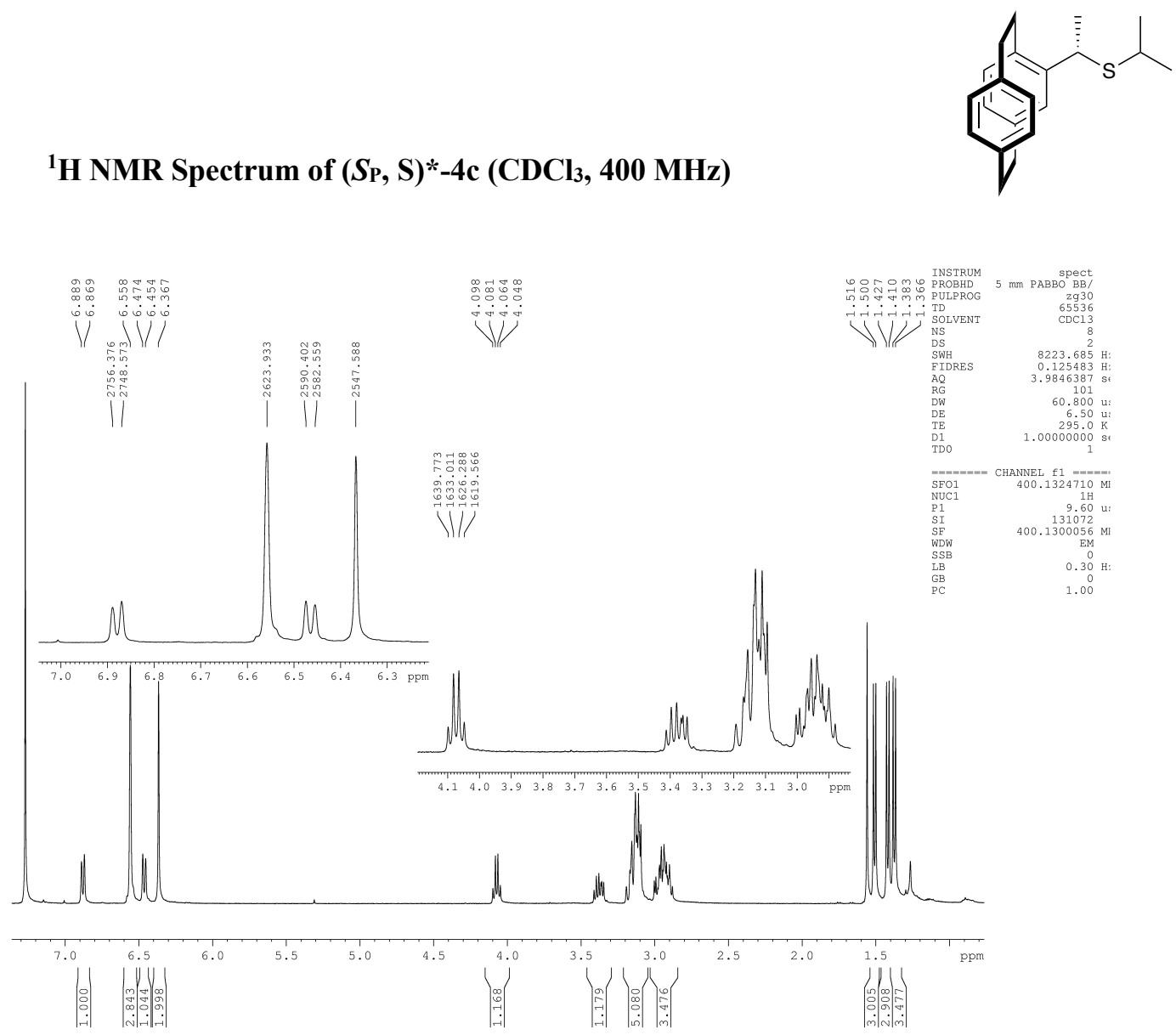

${ }^{13} \mathrm{C}\left\{{ }^{1} \mathrm{H}\right\}$ NMR Spectrum of $\left(S_{\mathrm{P}}, S\right)^{*}-4 \mathrm{c}\left(\mathrm{CDCl}_{3}, 101 \mathrm{MHz}\right)$

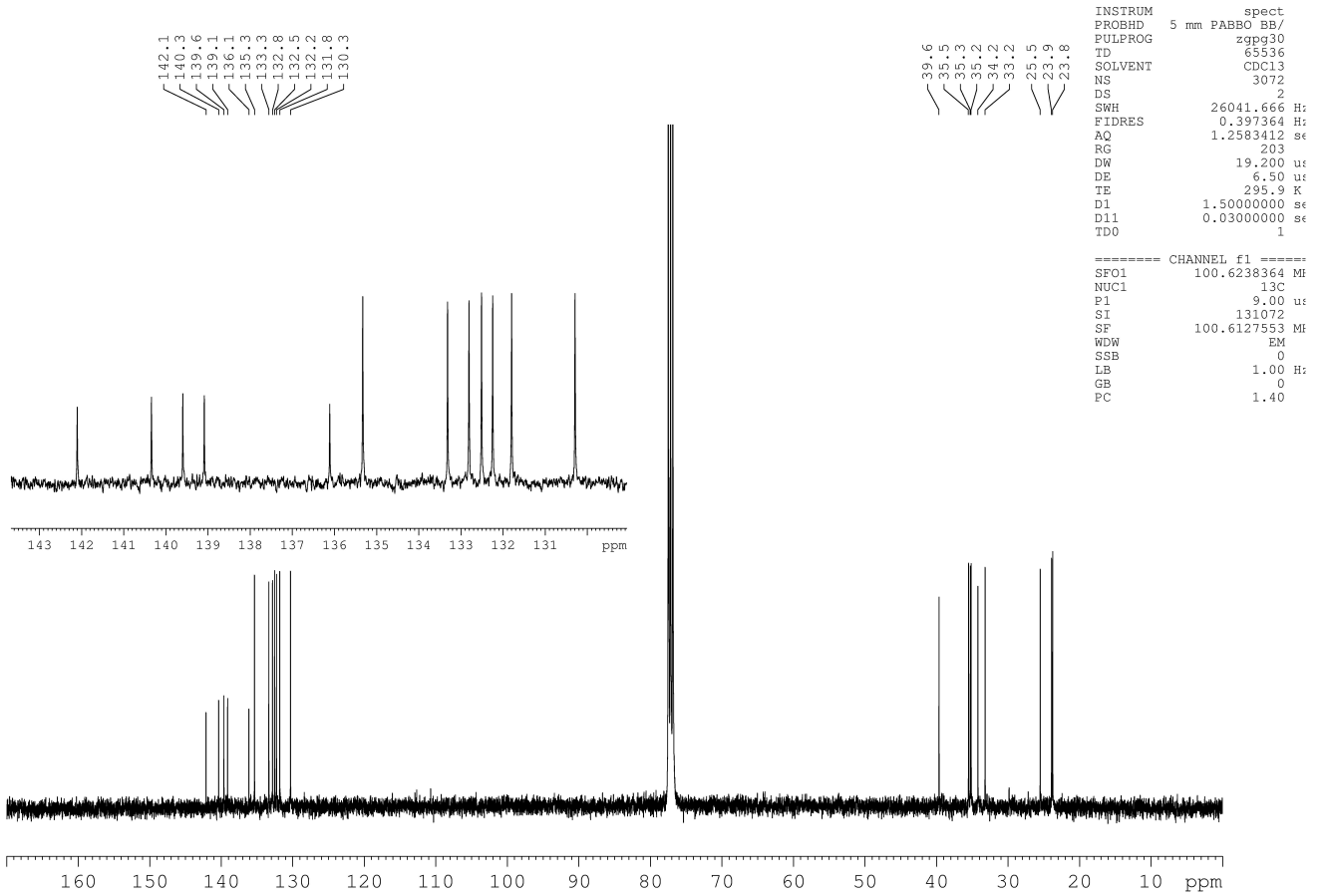


${ }^{1} \mathrm{H}$ NMR Spectrum of $5\left(\mathrm{CDCl}_{3}, 400 \mathrm{MHz}\right)$
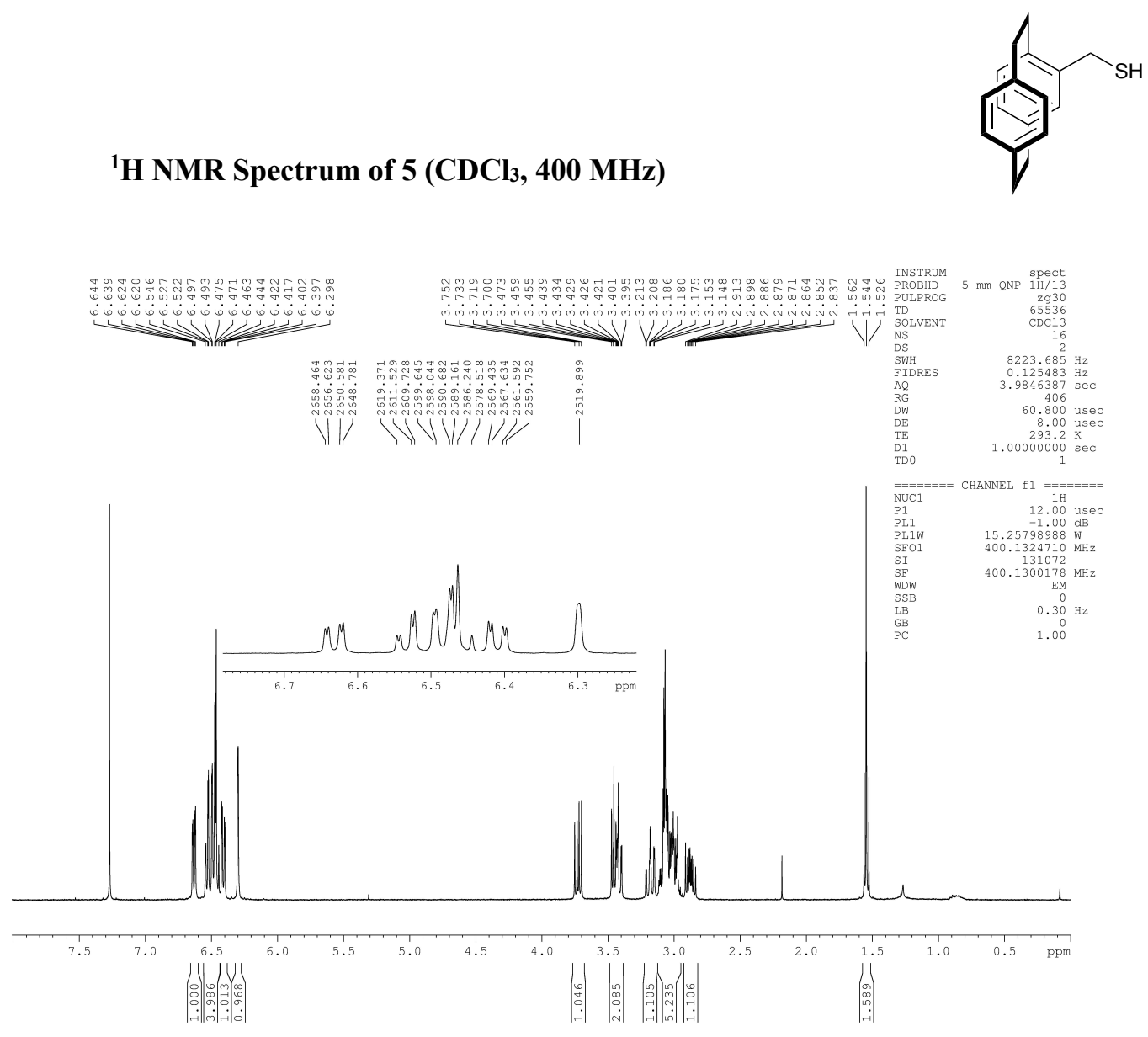

${ }^{13} \mathrm{C}\left\{{ }^{1} \mathrm{H}\right\}$ NMR Spectrum of $5\left(\mathrm{CDCl}_{3}, 101 \mathrm{MHz}\right)$

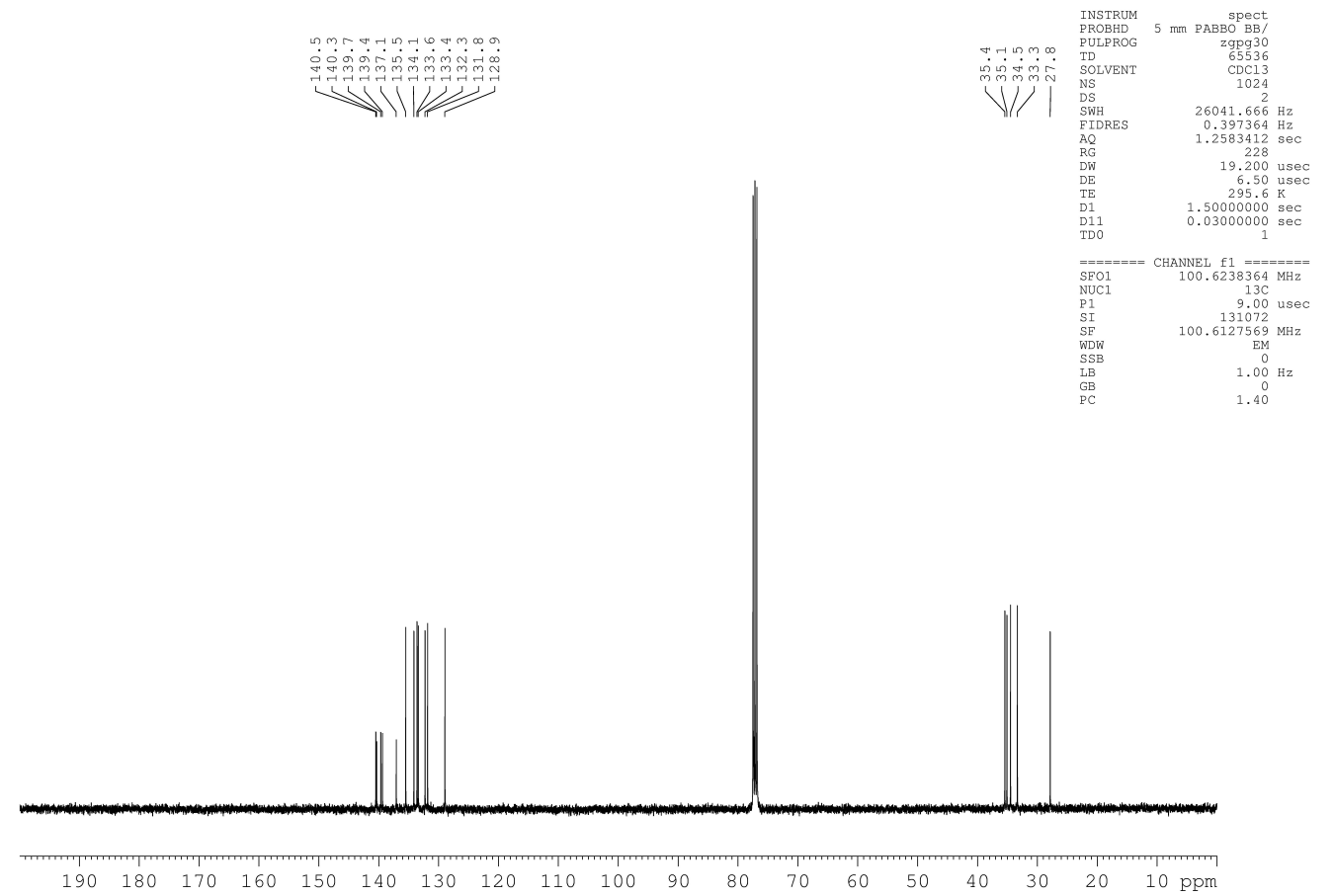

